\title{
Cryomicroneedles for transdermal cell delivery
}

\author{
Hao Chang ${ }^{1,2}$, Sharon W. T. Chew ${ }^{2}$, Mengjia Zheng ${ }^{2}$, Daniel Chin Shiuan Lio², Christian Wiraja², \\ Yu Mei ${ }^{3}$, Xiaoyu Ning ${ }^{2}$, Mingyue Cui ${ }^{2}$, Aung Than' ${ }^{2}$, Peng Shi ${ }^{1}$, Dongan Wang ${ }^{1}{ }^{1}$, Kanyi Pu ${ }^{2}$, \\ Peng Chen ${ }^{2}$, Haiyan Liu $\mathbb{1}^{3}$ and Chenjie Xu ${ }^{1,2}{ }^{1,}$
}

Cell therapies for the treatment of skin disorders could benefit from simple, safe and efficient technology for the transdermal delivery of therapeutic cells. Conventional cell delivery by hypodermic-needle injection is associated with poor patient compliance, requires trained personnel, generates waste and has non-negligible risks of injury and infection. Here, we report the design and proof-of-concept application of cryogenic microneedle patches for the transdermal delivery of living cells. The microneedles are fabricated by stepwise cryogenic micromoulding of cryogenic medium with pre-suspended cells, and can be easily inserted into porcine skin and dissolve after deployment of the cells. In mice, cells delivered by the cryomicroneedles retained their viability and proliferative capability. In mice with subcutaneous melanoma tumours, the delivery of ovalbumin-pulsed dendritic cells via the cryomicroneedles elicited higher antigen-specific immune responses and led to slower tumour growth than intravenous and subcutaneous injections of the cells. Biocompatible cryomicroneedles may facilitate minimally invasive cell delivery for a range of cell therapies.

T he skin provides crucial regulatory and defensive functions ${ }^{1,2}$. Skin disorders may put patients at risk of cutaneous and systemic infection, trauma and malignancies ${ }^{3}$. Although traditional therapeutics have limited capabilities to solve these problems, the latest advances in cell therapy bring hope for previously incurable and untreatable diseases ${ }^{4,5}$. For example, genetically modified epidermal stem cells can be transplanted to regenerate the skin in patients with epidermolysis bullosa ${ }^{6,7}$, and melanocytes can be transplanted to treat vitiligo ${ }^{8,9}$. Dendritic cell (DC)-based immunotherapy has proven to be a safe treatment for melanoma ${ }^{10,11}$. Currently, these therapeutic cells are delivered by either bolus injection for diseases of systemic health (for example, cancer) ${ }^{11}$ or scaffold transplantation for local symptoms (for example, eczema $)^{12}$. However, these methods have difficulty in achieving controlled and precise delivery of targeted cells without sacrificing the comfort of patients, which presents challenges for their translation to clinical applications.

Microneedle $(\mathrm{MN})$ technology is a promising option for realizing the controlled and precise delivery of targeted drugs into specific skin layers and other tissues (for example, mucosa and cornea) in a minimally invasive manner ${ }^{13-16}$. MNs are composed of a wide range of materials, geometries and spatial arrangements and function as carriers of small molecular drugs, peptides and proteins (for example, insulin, vaccines and antibodies), oligonucleotides and nanomedicines ${ }^{17,18}$. Unfortunately, none of these existing MN technologies can carry and deliver living formulations, such as therapeutic cells, into skin, without the assistance of extra devices. To carry and deliver cells, MNs have to maintain the viability of loaded cells and have mechanical strength to penetrate the skin.

Here, we introduce an MN patch that is specifically designed for packaging and delivering living cells into skin (Fig. 1). This MN patch, termed cryomicroneedles (cryoMNs), is fabricated by stepwise cryogenic micromoulding of the optimized cryogenic medium and pre-suspended cells of interest in the pre-designed
MN mould. CryoMNs can easily pierce skin and deliver loaded living cells into skin.

\section{Results}

Selection of cryogenic medium for the fabrication of cryoMNs. Ice is the solid state of water or an aqueous medium in a cryogenic environment and has strong mechanical properties ${ }^{19}$. Given that ice can be easily moulded to any shape, ice-based MNs can be fabricated easily in cryogenic environments. Furthermore, cells can be preserved in a cryogenic environment with minimal loss of viability if they are pre-suspended in a suitable cryoprotectant medium ${ }^{20,21}$.

Dimethyl sulfoxide (DMSO) is one of the most widely used cryoprotectant $\mathrm{s}^{22}$ and can minimize damage to cell membranes under a cryogenic environment by hindering the crystallization of ice ${ }^{21}$. We thus selected cryogenic medium supplemented with DMSO to fabricate the cryoMNs. We found that cryoMNs fabricated from cryogenic medium containing more than 2.5\% DMSO ( $\mathrm{vol} / \mathrm{vol}$ ) could not be peeled from the mould with their structures intact (observed as leftover rhodamine tips in the mould in Supplementary Fig. 1). However, a concentration of DMSO of less than $2.5 \%$ resulted in an obvious reduction in cell viability from $\sim 90$ to $\sim 10 \%$ (Supplementary Fig. 2).

To solve this dilemma, we incorporated a non-penetrating cryoprotectant into the medium to compensate for the loss of cell viability under low concentrations of $\mathrm{DMSO}^{23}$. In contrast to DMSO, non-penetrating cryoprotectants only act at the extracellular surface of cells and can reduce the amount of water in contact with cells by increasing the osmolarity of the medium ${ }^{24}$. In addition, DMSO pre-dehydrates cells at non-freezing temperatures (for example, room temperature) to their minimal volume with minimized osmotically active water, preventing cells from being injured during freezing ${ }^{23}$. As a proof of concept, we selected sucrose to combine with DMSO as the cryogenic medium. The viabilities of six types of human cell-HeLa cells expressing red fluorescent

'Department of Biomedical Engineering, City University of Hong Kong, Kowloon Tong, Hong Kong SAR, China. ${ }^{2}$ School of Chemical and Biomedical Engineering, Nanyang Technological University, Singapore, Singapore. ${ }^{3}$ Immunology Programme, Life Sciences Institute, Immunology Translational Research Programme and Department of Microbiology and Immunology, Yong Loo Lin School of Medicine, National University of Singapore, Singapore, Singapore. $\bowtie$ e-mail: chenjie.xu@cityu.edu.hk 


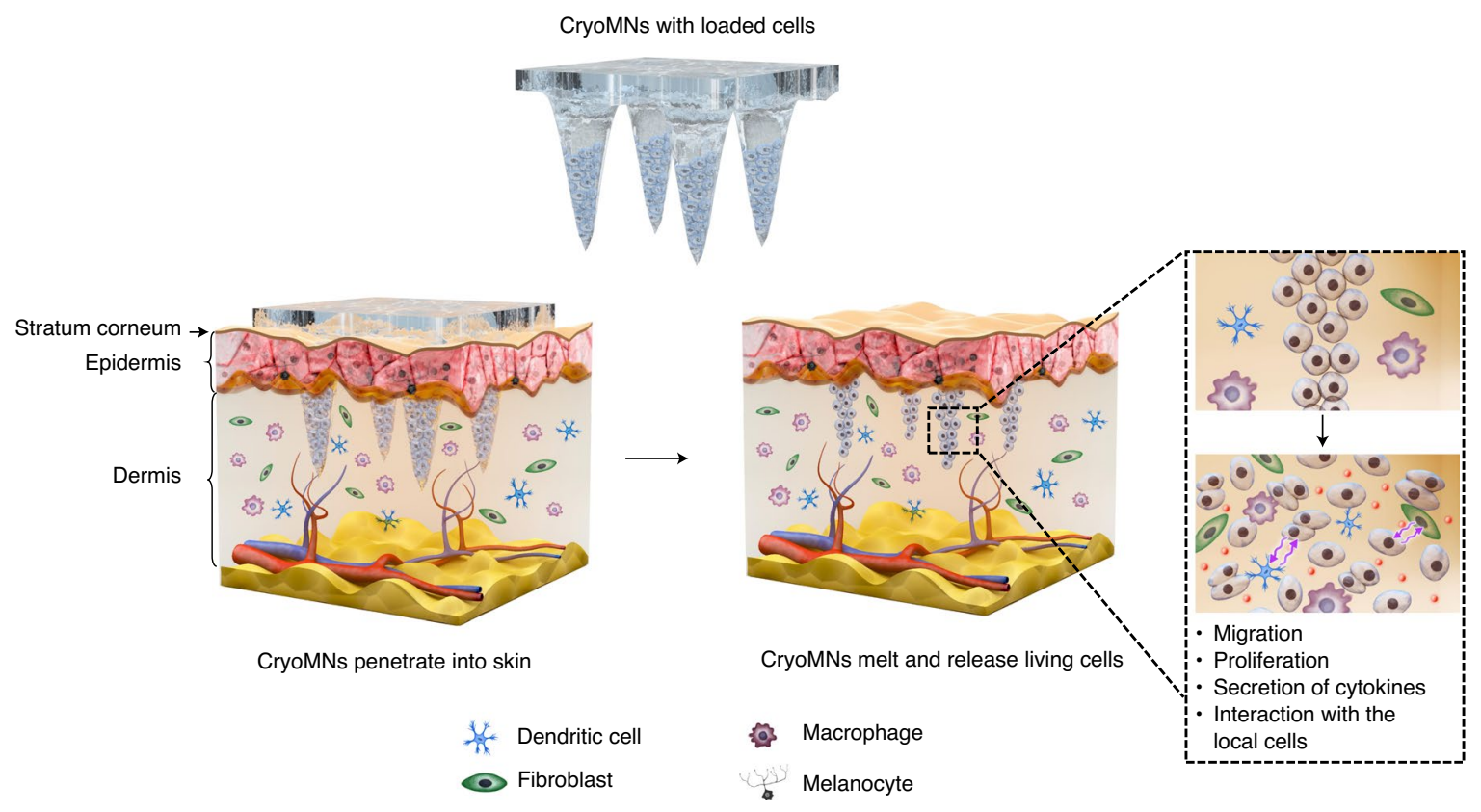

Fig. 1 | Schematic illustration of the transdermal delivery of cells using cryoMNs. The cryoMNs, loaded with cells (top), can easily penetrate into skin epidermis (bottom left). The loaded cells are released when the cryoMNs melt (right). The released cells subsequently migrate, proliferate, secrete specific cytokines and interact with other local cells in the skin (expanded panel).

protein (RFP-HeLa), keratinocytes (HaCaT), normal dermal fibroblasts (NDFs), mesenchymal stem cells (MSCs), epidermal melanocytes and peripheral blood CD8 ${ }^{+}$CD45RA ${ }^{+} \mathrm{T}$ cells ( $\mathrm{T}$ cells)-were evaluated after cryopreservation. As shown in Supplementary Fig. 2, regardless of the concentration, the addition of sucrose substantially improved cell viability, which was obvious when the concentration of DMSO was less than $5 \%$. This phenomenon was universal to all types of cell. No difference in cell viability was noted between $100 \mathrm{mM}$ and $200 \mathrm{mM}$ sucrose in $2.5 \%$ DMSO. In the following experiments, phosphate-buffered saline (PBS) supplemented with $2.5 \%$ DMSO and $100 \mathrm{mM}$ sucrose was used as the optimized cryogenic medium for fabricating cryoMNs. PBS was used as the base medium because it did not introduce other molecules (such as proteins) that might induce undesirable immune responses in practical applications.

Fabrication and characterization of cryoMNs. The cryoMNs were prepared using a micromoulding method (Fig. 2a), which is the easiest method with which to shape an aqueous solution into an MN structure. Cells of interest were pre-suspended in the optimized cryogenic medium, and the obtained mixture was cast into a negative polydimethylsiloxane (PDMS) mould. Therapeutic cells are typically rare and precious and it is desirable that they are not wasted during device fabrication, so, because the cryoMN base does not enter the skin layers, the cells need only be loaded into the tips of the cryoMNs. To ensure that the cells had entered the tips of the needles, the cells, suspended in the cryogenic medium, were left in the PDMS mould for 20 min without centrifugation to allow the cells to sink into the tips of the needles by gravity. During this 20 -min process, the sucrose simultaneously dehydrated the cells ${ }^{23}$. A sequential, gradient cryogenic process (from $-20^{\circ} \mathrm{C}$ to $-196^{\circ} \mathrm{C}$ ) was then applied, and the cryoMN patch was formed after solidification. The entire fabrication process took $\sim 8 \mathrm{~h}$.

The master template was a stainless-steel MN array $(10 \times 10)$, with each $\mathrm{MN}$ having a height of $1,200 \mu \mathrm{m}$ and a base width of $400 \mu \mathrm{m}$ (Supplementary Fig. 3). The resulting cryoMNs had a height of $\sim 900 \mu \mathrm{m}$ and a base width of $\sim 350 \mu \mathrm{m}$ (Fig. $2 \mathrm{~b}-\mathrm{d}$ ). The dimensional difference between the original template and cryoMNs was due to shrinking of the elastic PDMS during templating ${ }^{15}$. However, the pyramid structure and sharpness of the needles were well replicated. To visualize the loaded cells within cryoMNs, the model cells (HaCaT) were stained with Hoechst 33342 beforehand. After being immersed in cold PBS, the MN tips detached from the base. The stained cells were observed inside the needle (Fig. $2 \mathrm{e}-\mathrm{g}$ and Supplementary Fig. 4). Precipitation for $20 \mathrm{~min}$ allowed $\sim 80 \%$ of the cells to sink into the cavities (Supplementary Fig. 5), and $~ 800$ $\mathrm{HaCaT}$ cells were present in one MN tip (Methods). The cell capacity of a single $\mathrm{MN}$ depends on the dimension of the $\mathrm{MN}$ as well as the size of the cells. The capacities of the six types of cell used in this study ranged from $\sim 4 \times 10^{3}$ to $\sim 1.93 \times 10^{5}$ per single $\mathrm{MN}$ tip (Supplementary Fig. 6).

We also examined the mechanical strength of the cryoMNs using a tensile testing machine (Supplementary Video 1). Four types of polymeric $\mathrm{MN}$ that are commonly used for transdermal drug delivery (polystyrene MNs (PS-MNs), polylactic acid MNs (PLA-MNs), polycaprolactone MNs (PCL-MNs) and crosslinked hyaluronic acid MNs (MeHA-CL-MNs) ${ }^{15}$ ) were used for comparison. The compression strength was calculated from the slope of the stress-strain curve (Supplementary Fig. 7a,b). Specifically, the compressive strength of the cryoMNs was $24.7 \pm 3.8 \mathrm{MPa}$, which is comparable to the compressive strength of the PS-MNs $(66.2 \pm 10.9 \mathrm{MPa})$, PLA-MNs $(38.8 \pm 6.4 \mathrm{MPa}), \mathrm{PCL}-\mathrm{MNs}(17.6 \pm 4.1 \mathrm{MPa})$ and MeHA-CL-MNs $(17.7 \pm 3.0 \mathrm{MPa})$ (Fig. $2 \mathrm{~h})$. The load fracture force of the cryoMNs was $0.17 \mathrm{~N}$ (Supplementary Fig. 7a), which is greater than the minimum average force $(0.058 \mathrm{~N})$ required for normal skin penetration ${ }^{25}$.

Correlation between the skin penetration ability of cryoMNs and their residence time at room temperature. One potential challenge faced by the use of cryoMNs in practical applications is their limited lifetime, because ice will melt in the operating environment (typically at room temperature (RT)). The melting of cryoMNs definitely has a negative influence on their mechanical strength, which determines their skin penetration ability. When the cryoMNs were removed from their cryopreservation environment $\left(-196^{\circ} \mathrm{C}\right.$, 

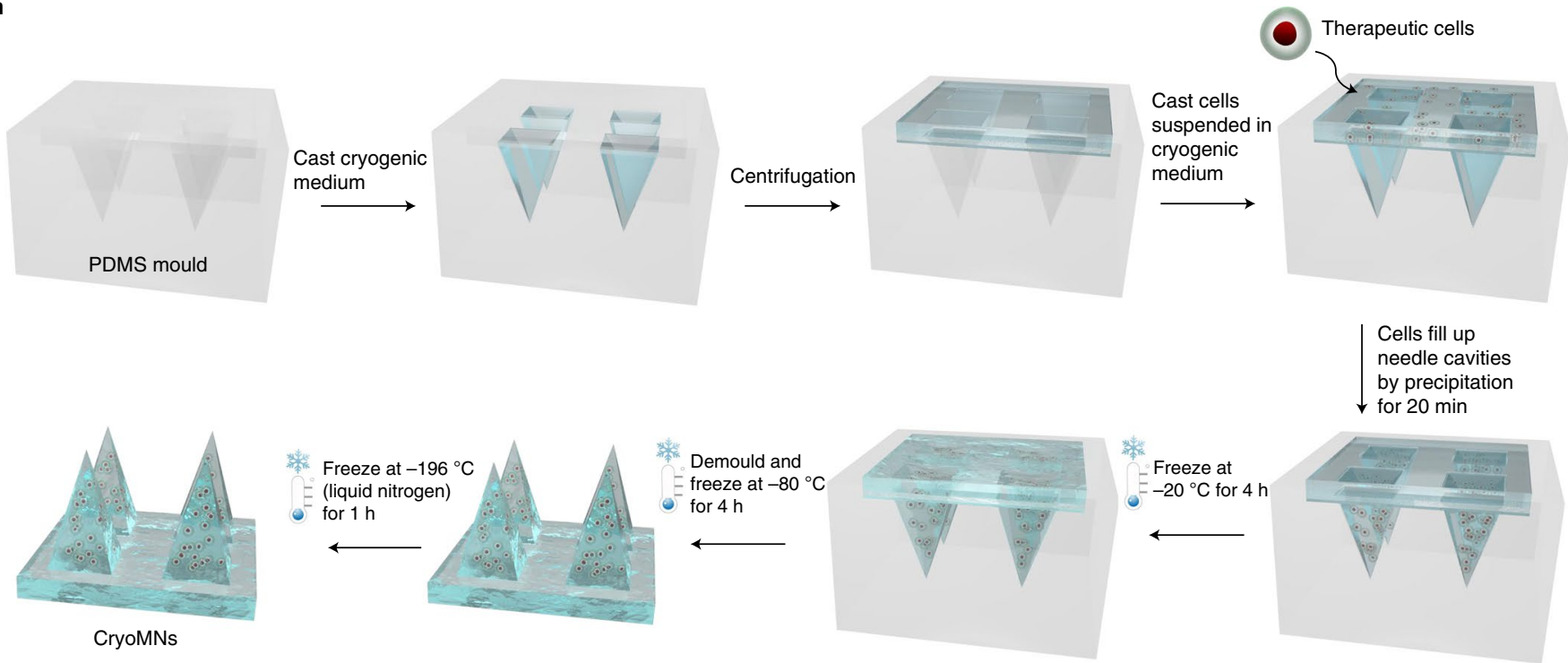

CryoMNs
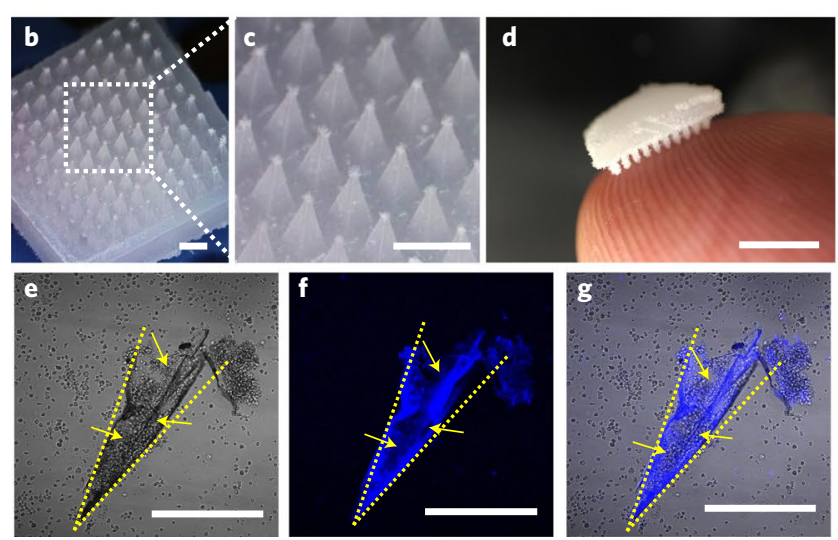

h
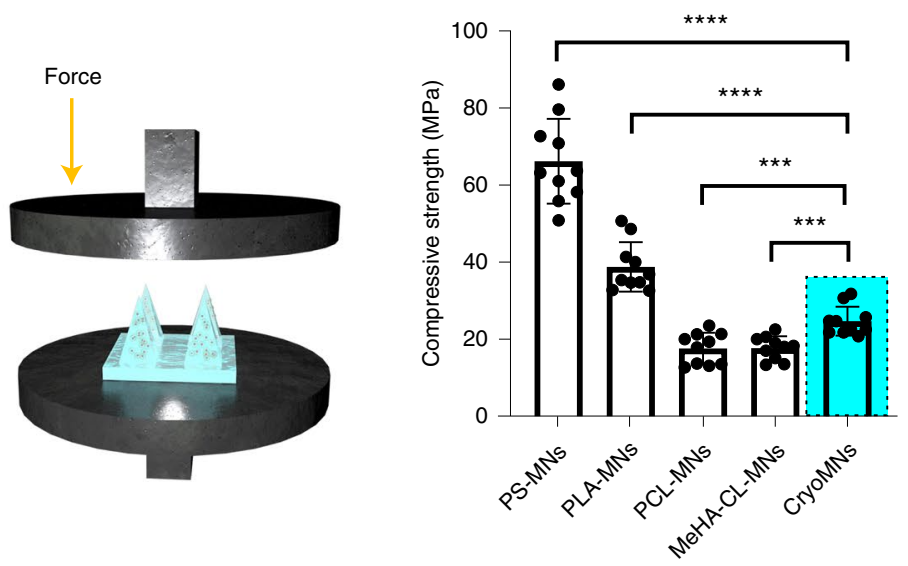

Fig. 2 | Fabrication and characterization of cryoMNs. a, Schematic of cryoMN fabrication. b, Digital microscopy image of the cryoMNs. Scale bar, $1,000 \mu \mathrm{m}$. c, Magnified view of $\mathbf{b}$. Scale bar, 1,000 $\mu \mathrm{m}$. d, Photograph of the cryoMNs patch. Scale bar, $5 \mathrm{~mm}$. e-g, Images of a single MN loaded with cells stained with NucBlue Live ReadyProbes reagent. Yellow arrows indicate individual cells. Image $\mathbf{g}$ is an overlay of the bright-field image in $\mathbf{e}$ and a fluorescent image (f). Scale bars, $500 \mu \mathrm{m}$. h, Compressive strength of cryoMNs (right) tested by an Instron tensile meter (left). The blue area bounded by the dotted line highlights the cryoMNs. PS-MNs, polystyrene MNs; PLA-MNs, polylactic acid MNs; PCL-MNs, polycaprolactone MNs; MeHA-CL-MNs, crosslinked hyaluronic acid MNs. Data are presented as mean \pm s.d. ( $n=10$ independent cryoMN samples). Two-tailed Student's $t$-test was used for comparing cryoMNs with other conventional polymeric microneedles ( ${ }^{\star \star \star \star} P=1.31972 \times 10^{-9}$ versus PS-MNs; ${ }^{\star \star \star \star} P=1.15268 \times 10^{-5}$ versus $\mathrm{PLA}-\mathrm{MNs}$; ${ }^{\star \star \star} P=0.0008$ versus $\mathrm{PCL}-\mathrm{MNs}$; ${ }^{\star \star} \mathrm{P}=0.0002$ versus $\mathrm{MeHA}-\mathrm{CL}-\mathrm{MNs}$ ). In $\mathbf{e}-\mathbf{g}$, three images were taken and all experiments were repeated independently twice, with similar results.

liquid nitrogen) and left at RT (in this case RT was $\sim 24^{\circ} \mathrm{C}$ ), frost appeared immediately on the cryoMNs (Supplementary Fig. 8a and Supplementary Video 2). After 140 s, the needle tips began to melt. When the cryoMNs were placed on a fingertip, the needle tips melted in 60 s (Supplementary Fig. 8b and Supplementary Video 3).

The correlation between skin penetration ability and the residence time at RT was examined with porcine skin, given its recognized similarities to the anatomy of human $\operatorname{skin}^{26}$. As shown in Fig. 3a,b, the skin penetration ability decreased when the cryoMNs were exposed to RT for longer durations, and they lost their skin penetration ability if they stayed at RT for more than $50 \mathrm{~s}$. At a residence time of $40 \mathrm{~s}$, the cryoMNs could pierce through the stratum corneum, which is $\sim 21-26 \mu \mathrm{m}$ thick $^{26}$. The cryoMNs incubated at RT for less than $30 \mathrm{~s}$ were able to reach the dermal layer (up to $516 \pm 76 \mu \mathrm{m}$ ). These results indicate that cryoMNs should be administered to patients immediately after being removed from the storage environment and that the exposure times to RT should be less than 40 s (Fig. 3a,b and Supplementary Fig. 9). During the experiment, we found that this time was sufficient for the successful penetration of cryoMNs.

As a proof of concept, we delivered RFP-HeLa cells into porcine skin by using cryoMNs with a 10 -s residence time. Both nuclear staining and RFP signals confirmed the presence of cells in the dermis layer (Fig. 3c). Similar to the observation in Fig. 3a, a prolonged residence time decreased the skin penetration ability of cryoMNs and the depth that the cells could reach (Supplementary Figs. 10 and 11). CryoMNs could be stored at both $-196^{\circ} \mathrm{C}$ and $-80^{\circ} \mathrm{C}$, which is similar to common cryopreservation conditions. However, at RT, the cryoMNs stored at $-80^{\circ} \mathrm{C}$ melted more quickly than those stored at $-196^{\circ} \mathrm{C}$ (Supplementary Video 4) and had to be applied within $20 \mathrm{~s}$ for successful skin penetration (Supplementary Figs. 9 and 12). 
a
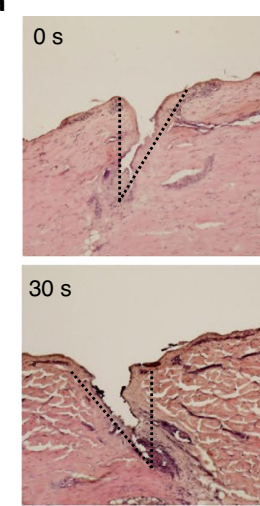

C

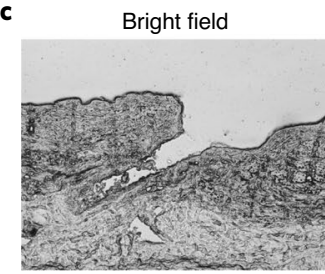

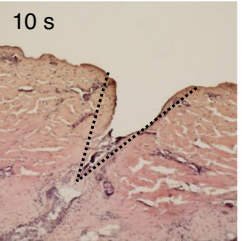

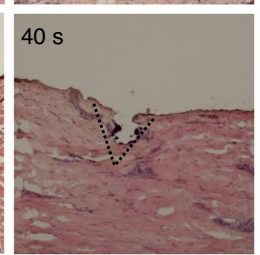

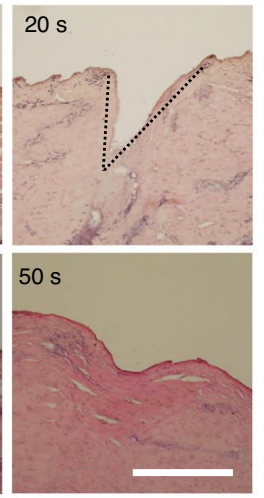

b

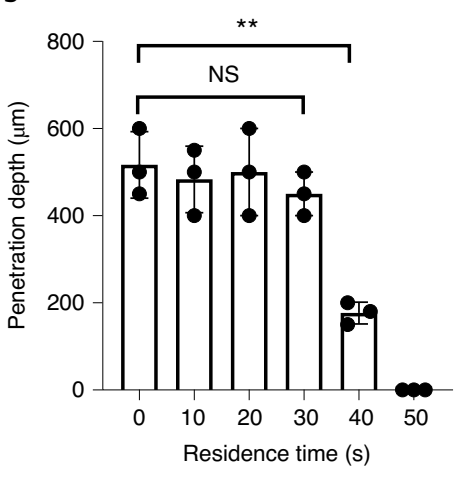

RFP

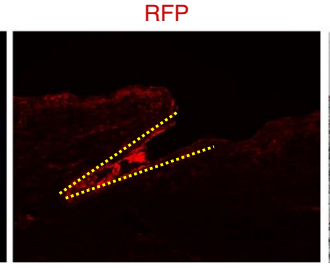

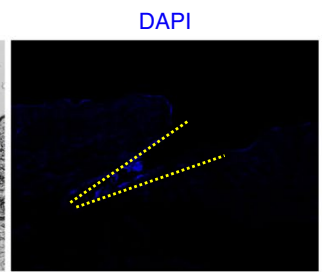

Fig. 3 | Correlation between the skin penetration ability of cryoMNs and their residence time at RT. a, Representative haematoxylin and eosin (H\&E) staining images of porcine skin inserted by cryoMNs. The cryoMNs were removed from their cryopreservation environment ( $-196^{\circ} \mathrm{C}$, liquid nitrogen) and placed at RT $\left(24^{\circ} \mathrm{C}\right)$ for $0,10,20,30,40$ and $50 \mathrm{~s}$ before skin penetration. Scale bar, $500 \mu \mathrm{m}$. b, Quantitative data for the influence of residence time on skin penetration depth. Data are presented as mean \pm s.d. ( $n=3$ independent cryoMN samples). Two-tailed Student's $t$-test was used to compare the penetration depth after a residence time of 0 s and residence times of 30 s and 40 s (NS, $P=0.2746$ versus 30 s; ${ }^{\star \star} P=0.0019$ versus 40 s). c, Fluorescent images of skin cryosection after application of RFP-HeLa-loaded cryoMNs. The cell nucleus was stained with NucBlue Live ReadyProbes reagent in advance. The needle shape is outlined with dashed lines. Scale bar, $200 \mu \mathrm{m}$. In a and c, three images were taken in each experiment and all experiments were repeated independently twice, with similar results.

Survival and proliferation of cells released from cryoMNs. The six types of cell (RFP-HeLa, HaCaT, NDFs, MSCs, melanocytes and $\mathrm{T}$ cells) were loaded into cryoMNs with the above-mentioned fabrication process. Cell viability was tested after melting these fresh cryoMNs in PBS $\left(37^{\circ} \mathrm{C}\right)$. According to live/dead staining (Fig. $4 \mathrm{a}$ and Supplementary Fig. 13), viability varied among the different types. Specifically, the viability was $38.7 \pm 2.7 \%$ for RFP-HeLa cells, $34.8 \pm 6.5 \%$ for $\mathrm{HaCaT}$ cells, $30.0 \pm 2.4 \%$ for $\mathrm{NDF}$, $30.8 \pm 2.2 \%$ for MSCs, $53.1 \pm 2.4 \%$ for melanocytes and $21.4 \pm 2.2 \%$ for $\mathrm{T}$ cells (Fig. 4b). In addition, these living cells could proliferate after being released from cryoMNs (Fig. 4c and Supplementary Fig. 14). At six days, the cell population became 13.8-fold for RFP-HeLa cells, 8.5-fold for HaCaT cells, 2.0-fold for NDFs, 3.1-fold for MSCs, 1.7-fold for melanocytes and 1.9-fold for $\mathrm{T}$ cells. These data were similar to the proliferation of cells without any processing (Supplementary Fig. 15), suggesting that the fabrication process did not influence cell proliferation. In addition, there was minimal change in the viability of RFP-HeLa cells and melanocytes in cryoMNs after being preserved in a cryogenic environment for one month (Supplementary Fig. 16), suggesting that the cryoMNs are suitable for long-term storage. In addition, the viability and proliferation ability of cells in cryoMNs from $-80^{\circ} \mathrm{C}$ were similar to those of cells in cryoMNs from $-196^{\circ} \mathrm{C}$ (Fig. $4 \mathrm{a}-\mathrm{C}$ and Supplementary Fig. 17). Furthermore, a three-dimensional (3D) skin model was established by coating agarose hydrogel (as dermis) with a parafilm layer (as epidermis), as in a previous study ${ }^{15}$. After being delivered into the skin model with cryoMNs, MSCs were able to proliferate within 12 days (Supplementary Fig. 18).

Next, RFP-HeLa-loaded cryoMNs were applied on the back of mouse skin through a thumb press (Fig. $4 \mathrm{~d}-\mathrm{f}$ ). Fluorescent imaging of the skin cryosections (Fig. $4 \mathrm{~g}$ and Supplementary Fig. 19) revealed that RFP-HeLa cells were present in the dermis with depths ranging from $\sim 20$ to $200 \mu \mathrm{m}$ (the thickness of the mouse epidermis is $\sim 15 \mu \mathrm{m})^{27}$. Blank cryoMNs and RFP-HeLa-loaded cryoMNs were applied on two sides of the mouse back to monitor the fluorescent signals in mouse skin (Fig. 4h). Following transplantation, the RFP signal was consistently observed on the sites treated with RFP-HeLa-loaded cryoMNs, whereas no signal was present on the sites treated with blank cryoMNs (Fig. 4h,i). After one day of implantation, the fluorescence intensity was reduced $\sim 75 \%$ compared with that on day 1 , which should be due to the death of some cells at the initial implantation and the loss of the cells that were on the surface of the skin. The implanted cells displayed slight proliferation from day 3 to day 14 and continued to maintain viability over 14 days inside the skin of the mice (Fig. 4h,i).

Vaccination by cryoMNs carrying antigen-loaded DCs. DCs are important antigen-presenting cells, and DC vaccines have been widely applied in immunotherapy for cancer treatment ${ }^{11,28,29}$. Skin is recognized as an ideal target site for vaccination because skin is a highly immunocompetent tissue containing a large population of resident antigen-presenting cells ${ }^{30}$. Here, we evaluated the therapeutic effect of DC-loaded cryoMNs for cancer vaccination as a proof of concept. Specifically, bone marrow-derived DCs were pulsed with $50 \mu \mathrm{g} \mathrm{ml}^{-1}$ ovalbumin (OVA; as a model antigen) to obtain OVA-pulsed DCs (OVA-DCs), which were loaded into cryoMNs (Fig. 5a). The levels of the surface marker CD86 and major histocompatibility complex class II (MHCII) were used to confirm the activation and maturation of DCs (Supplementary Fig. 20a,b). Lipopolysaccharide (LPS)-treated DCs (LPS-DCs) served as a positive control. The viability of the OVA-DCs in cryoMNs was $71.4 \pm 1.4 \%$ (Supplementary Fig. 20b,c). In addition, the viability of the OVA-DCs in cryoMNs did not change even after one month of storage in liquid nitrogen (Supplementary Figs. 20c and 21). 

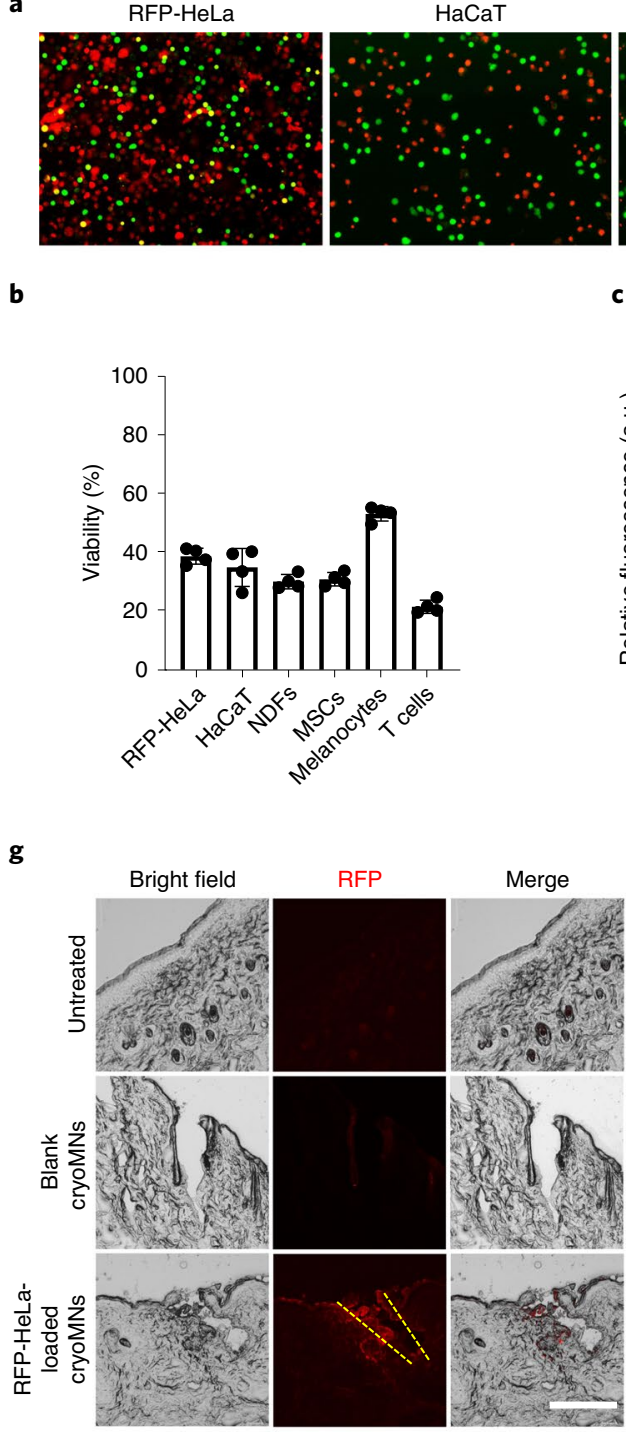

NDFs

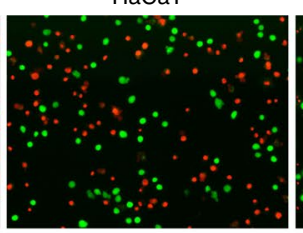

MSCs

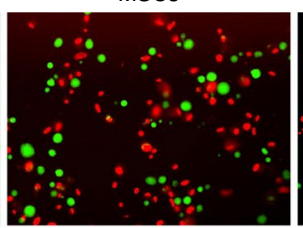

Melanocytes

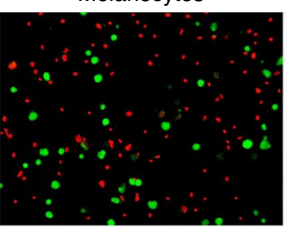

T cells

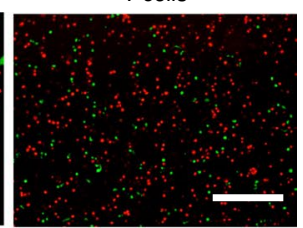

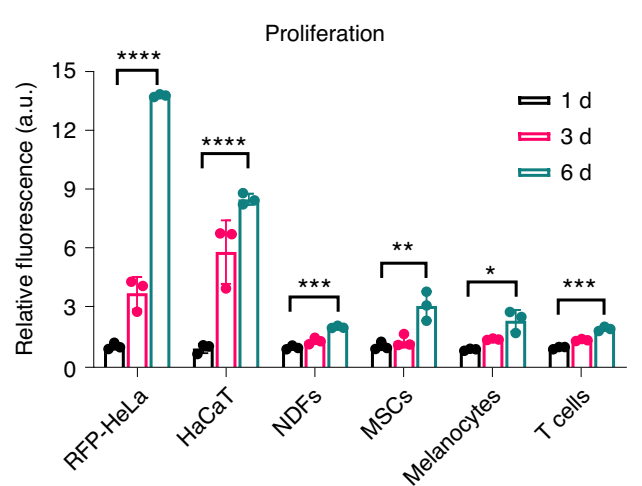

d

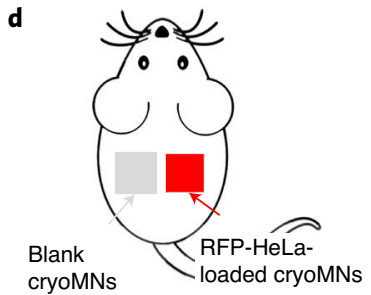

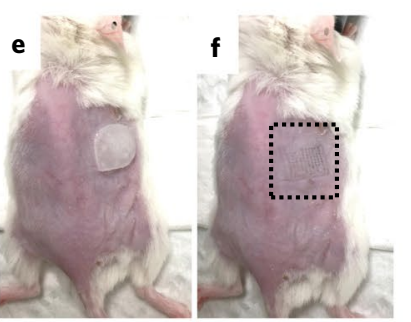
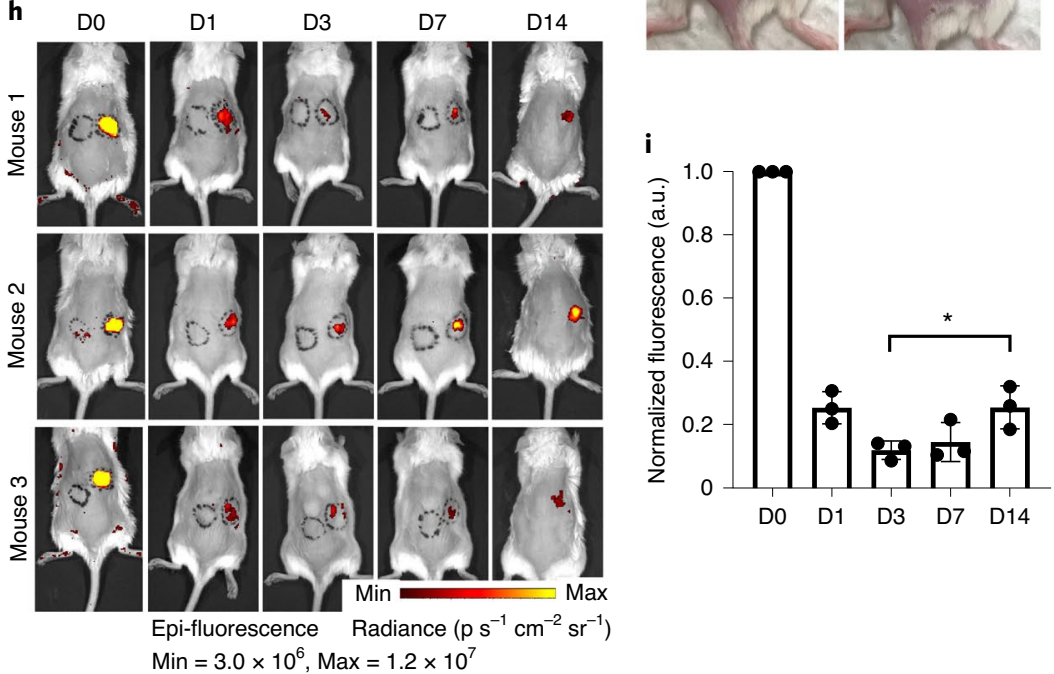

Fig. 4 | Viability and proliferation of cells released from the cryoMNs after being removed from their cryopreservation environment ( $-196^{\circ} \mathrm{C}$, liquid nitrogen). a, Live (green)/dead (red) staining images of the cells loaded in cryoMNs. Scale bar, $200 \mu \mathrm{m}$. Four images were taken for each group. All experiments were repeated independently three times, with similar results. b, Quantitative data of cell viability calculated from live (green)/dead (red) staining by dividing the number of living cells (green) by the total number of cells. Data are presented as mean \pm s.d. ( $n=4$ biologically independent samples). c, Proliferation of the cells after being released from cryoMNs and cultured for one, three and six days. Data were obtained by using the alamarBlue assay, and the fluorescence intensity obtained on the first day was used as reference. Data are presented as mean \pm s.d. ( $n=3$ biologically independent samples). Two-tailed Student's $t$-test was used for comparing the relative fluorescence at day 6 with the relative fluorescence at day 1 in each group $\left({ }^{\star \star \star \star} P=2.37951 \times 10^{-8}\right.$ for RFP-HeLa; ${ }^{\star \star \star \star} P=3.94959 \times 10^{-6}$ for HaCaT; ${ }^{\star \star \star} P=0.0001$ for NDFs; ${ }^{\star \star} P=0.0096$ for MSCs; $P=0.0104$ for melanocytes; ${ }^{* \star *} P=0.0002$ for T cells). d, Schematic of the application of blank cryoMNs and RFP-HeLa-cell-loaded cryoMNs on the backs of mice. e,f, Photographs of the mouse skin during (e) and after (f) application of cryoMNs. The injection site is bounded by the black dotted square. $\mathbf{g}$, Fluorescent images of cryosections of untreated mouse skin and skin treated with blank cryoMNs and RFP-HeLa-cell-loaded cryoMNs. Skin can induce very light autofluorescence. Scale bar, $200 \mu \mathrm{m}$. Two images were taken for each group and all experiments were repeated independently twice, with similar results. $\mathbf{h}$, In vivo fluorescent imaging of mice treated with blank cryoMNs (black circles on the left of each mouse) and RFP-HeLa-cell-loaded cryoMNs (black circled area on the right side of each mouse) on days 0, 1, 3, 7 and 14. i, Quantification of fluorescence intensity on the skins. Data are normalized to the fluorescence intensity at day 0. Data are presented as mean \pm s.d. ( $n=3$ independent animals). Two-tailed Student's $t$-test was used for comparing the fluorescence at day 3 with the fluorescence at day $14\left({ }^{\star} P=0.0335\right)$.

We first optimized the administration frequency of OVA-DC-loaded cryoMNs (OVA-DC-cryoMNs) for cancer vaccination in mice. The mice received intradermal vaccination with four patches of OVA-DC-cryoMNs $\left(1 \times 10^{5}\right.$ OVA-DCs per patch) once per week, twice per week and three times per week over a four-week period (Supplementary Fig. 22a). At day 28, the draining lymph nodes (dLNs) were excised, and the maturation of DCs was examined based on the expression of co-stimulatory molecules, including MHCII and CD86. Mice receiving two vaccinations per week exhibited a higher percentage of CD11 $c^{+} \mathrm{CD} 86^{+} \mathrm{DCs}(4.21 \pm 0.16 \%)$ 
and $\mathrm{CD} 11 \mathrm{c}^{+} \mathrm{MHCII}^{+} \mathrm{DCs}(4.09 \pm 0.10 \%)$ in the dLNs than the other groups (Supplementary Fig. 22b-d). Splenocytes extracted from mice treated twice per week and three times per week showed better proliferation and higher secretion of interferon gamma (IFN- $\gamma$ ) after restimulation with OVA (Supplementary Fig. 22e,f). These data indicate that vaccination with OVA-DC-cryoMNs twice per week is adequate to induce an immune response.

Next, the optimal dosage was assessed. Following the optimized frequency of vaccination, the mice were administered two patches $\left(2 \times 10^{5}\right.$ OVA-DCs in all $)$, four patches $\left(4 \times 10^{5}\right.$ OVA-DCs in all $)$ and six patches $\left(6 \times 10^{5}\right.$ OVA-DCs in all $)$ of OVA-DC-cryoMNs over the same four-week period (twice per week) (Supplementary Fig. 23a). Analysis of excised dLNs at day 28 revealed that mice vaccinated with $4 \times 10^{5}$ OVA-DCs resulted in a higher percentage of CD11 $\mathrm{c}^{+} \mathrm{CD} 86^{+}$ DCs $(6.75 \pm 0.08 \%)$ and CD11 ${ }^{+} \mathrm{MHCII}^{+}$DCs $(7.82 \pm 0.13 \%)$ than the other groups (Supplementary Fig. 23b-d). In addition, vaccination with $4 \times 10^{5}$ OVA-DCs and $6 \times 10^{5}$ OVA-DCs via cryoMNs induced better proliferation of splenocytes and higher secretion levels of IFN- $\gamma$ compared to the other groups (Supplementary Fig. 23e,f). According to these results, a dosage of $4 \times 10^{5}$ OVA-DCs was selected as the optimal dosage, which involves fewer cells without compensation for the therapeutic effect.

After identifying the optimal administration frequency (twice per week) and dosage $\left(4 \times 10^{5}\right.$ OVA-DCs), we compared the therapeutic efficacy of vaccination with OVA-DC-cryoMNs to conventional vaccination methods, namely, s.c. and i.v. injection of OVA-DCs (Fig. 5b). For vaccination with OVA-DC-cyroMNs, each mouse was treated with four patches (that is, the optimized dosage) during one vaccination (Fig. 5 c). On day 28 , vaccination with OVA-DC-cryoMNs induced $3.59 \pm 0.26 \% \mathrm{CD}^{\circ} 1 \mathrm{c}^{+} \mathrm{CD} 86^{+} \mathrm{DCs}$ and $3.60 \pm 0.64 \% \mathrm{CD} 11 \mathrm{c}^{+} \mathrm{MHCII}^{+} \mathrm{DCs}$ in the dLNs, and these values greatly increased compared with vaccination by s.c. $(2.55 \pm 0.37 \%$ and $2.64 \pm 0.35 \%$, respectively) and i.v. injection $(2.46 \pm 0.68 \%$ and $2.40 \pm 0.45 \%$, respectively) (Fig. $5 \mathrm{~d}-\mathrm{f}$ ). After two days of culture, splenocytes from mice vaccinated with OVA-DC-cryoMNs displayed faster proliferation (Fig. 5g) and secreted higher levels of IFN- $\gamma$ (Fig. 5h) compared with those from mice vaccinated with s.c. and i.v. injection. The OVA-specific cytotoxic T lymphocyte (CTL) lysis of splenic T lymphocytes from vaccinated mice to the B16 melanoma cell line transfected with ovalbumin (B16-OVA) depended on the ratio of the effector and target cells (Fig. 5i). OVA-DC-cryoMNs induced significantly greater lysis efficiency than s.c. and i.v. injection of OVA-DCs (Fig. 5i). Collectively, vaccination with OVA-DC-cryoMNs could induce more potent antigen-specific immune responses than vaccination with s.c. and i.v. injection.

Finally, we studied the efficacy of tumour prevention of vaccination with OVA-DC-cryoMNs by inoculating mice with B16-OVA cells four days after the final vaccination. All groups vaccinated with
OVA-DCs displayed obviously delayed tumour growth (Fig. 5j). On day 26 post tumour inoculation, the tumours from the group vaccinated with OVA-DC-cryoMNs were much smaller, with a volume of $133.3 \pm 18.8 \mathrm{~mm}^{3}$ and weight of $57.8 \pm 23.7 \mathrm{mg}$, compared to those from the groups with s.c. injection $\left(240.7 \pm 24.6 \mathrm{~mm}^{3}\right.$ and $141.1 \pm 24.3 \mathrm{mg})$ and i.v. injection $\left(375.37 \pm 95.2 \mathrm{~mm}^{3}\right.$ and $137.4 \pm 9.4 \mathrm{mg}$ ) (Fig. 5k,1 and Supplementary Fig. 24). This finding suggests that vaccination with OVA-DC-cryoMNs displayed notably stronger antitumorigenic ability compared with vaccination with two conventional standard methods, namely, s.c. and i.v. injection.

Safety and biocompatibility evaluation. To study the biocompatibility of the cryoMNs, they were inserted into the back skin of mice through a thumb press and removed after $10 \mathrm{~min}$. A conventional polymeric MN patch made of hyaluronic acid (HA) (HA-MNs) was applied onto the skin of the same mouse for comparison. HA-MN patches have been widely used for the transdermal delivery of therapeutic agents due to their recognized biocompatibility and safety ${ }^{18,31}$. After removal, no obvious skin damage was noted at the administration sites of either cryoMNs or HA-MNs. The microholes were clearly observed immediately after application but became invisible within $10 \mathrm{~min}$ and completely disappeared after $30 \mathrm{~min}$ (Fig. 6a). This fast resealing of skin is essential to prevent the entry of pathogenic microbes or any toxic substances and further reduce the risk of infection. In addition, skin treated with both cryoMNs and HA-MNs did not exhibit any obvious erythema or oedema (Fig. 6a).

$\mathrm{H} \&$ Estaining revealed no obvious inflammatory cell infiltration or pathophysiological response at $30 \mathrm{~min}$ and $24 \mathrm{~h}$ post-administration of cryoMNs (Fig. 6b and Supplementary Fig. 25). In addition, we stained the skin tissue with the in situ terminal deoxyribonucleotidyl transferase (TDT)-mediated dUTP-digoxigenin nick end labelling (TUNEL) assay and did not observe noticeable cell apoptosis in the skin treated with either cryoMNs or HA-MNs (Fig. $6 \mathrm{c}$ and Supplementary Fig. 26).

Finally, frequent administration of cryoMNs neither induced any obvious hepatic damage (Fig. 6d) nor resulted in any weight loss in the mice (Fig. 6e). All the major organs looked normal after application of cryoMNs (Supplementary Fig. 27).

\section{Discussion}

Traditional cell delivery methods mainly include surgical intervention $^{32}$ and injection with a hypodermic needle ${ }^{33}$. These methods are associated with considerable disadvantages, including pain or discomfort, scarring, potential risk of infection, stick injuries from sharp waste and the need for skilled healthcare professionals. By contrast, cell delivery with cryoMNs is minimally invasive, generates no sharp hazard, and can be performed by end users with minimal expertise. In $\mathrm{MN}$-based technologies, the packaging and delivery of living therapeutic formulations is one of the most

Fig. $\mathbf{5}$ | Vaccination with OVA-DC-loaded cryoMNs (OVA-DC-cryoMNs). a, Schematic of loading OVA-DCs in cryoMNs. b, The protocol of vaccination with OVA-DC-cryoMNs in mice. Vaccinations with subcutaneous (s.c.) and intravenous (i.v.) injections of OVA-DCs were used for comparison. c, Photograph of the administration sites of OVA-DC-cryoMNs on the back skin of mice (left) and an enlarged photograph of the same mouse (right). The sites are bounded

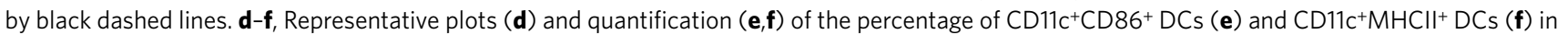
draining lymph nodes (dLNs) excised from mice in different treatment groups, analysed by flow cytometry. $\mathbf{g}$, In vitro proliferation of extracted splenocytes restimulated with $50 \mu \mathrm{g} \mathrm{ml}^{-1}$ antigen (OVA). The fluorescence intensity was obtained using an alamarBlue assay. $\mathbf{h}$, Secretion level of IFN- $\gamma$ in the culture supernatants after $48 \mathrm{~h}$ of culture. i, Determination of cytotoxic T lymphocyte (CTL) activity in vitro. Effector cells (splenocytes) and target cells (B16-OVA cells) were co-cultured at different ratios. 'E:T' refers to the ratio of effector cells (E) and target cells $(T)$. $\mathbf{j}$, Average tumour growth of mice bearing established B16-OVA melanoma tumours after different vaccination treatments. $\mathbf{k}, \mathbf{l}$, Weight (k) and photographs (I) of excised tumours at day 26 from five different mice (left to right) in each treatment group. In $\mathbf{e}-\mathbf{k}$, data are presented as mean \pm s.d. ( $n=5$ independent animals). Two-tailed Student's $t$-test was used to compare vaccination with OVA-DC-cryoMNs with s.c. injection and i.v. injection. In $\mathbf{e}^{\star \star \star \star} P=0.0009$ versus s.c. injection, ${ }^{\star \star} P=0.0084$ versus i.v. injection. In $\mathbf{f},{ }^{\star} P=0.0160$ versus s.c. injection, ${ }^{\star \star} P=0.0091$ versus i.v. injection. In $\mathbf{g},{ }^{\star \star \star \star} P=2.54937 \times 10^{-6}$ versus s.c. injection, ${ }^{\star \star \star \star} P=2.51656 \times 10^{-6}$ versus i.v. injections. In $\mathbf{h},{ }^{\star \star \star} P=0.0003$ versus s.c. injection, ${ }^{\star \star \star \star} P=2.19911 \times 10^{-6}$ versus i.v. injection. In $\mathbf{i}$, at E:T $=100: 1,{ }^{\star \star \star} P=0.0009$ versus s.c. injection, ${ }^{\star \star \star} P=0.0004$ versus i.v. injection. In $\mathbf{j}$, at day $26,{ }^{\star \star \star \star} P=5.50942 \times 10^{-5}$ versus s.c. injection, ${ }^{\star \star \star} P=0.0005$ versus i.v. injection. In $\mathbf{k},{ }^{\star \star \star} P=0.0006$ versus s.c. injection, ${ }^{\star \star \star} P=0.0001$ versus i.v. injection. 
important applications. For example, Bacille Calmette-Guérin (BCG) bacilli are loaded in dry MN patches as vaccines for tuberculosis prevention $^{34,35}$. Cardiac stromal cells (CSCs) are integrated with the back patch of MNs, which serve as pathways allowing regenerative factors secreted by the CSCs to be released into the injured myocardium ${ }^{36}$. However, all these existing $\mathrm{MN}$ technologies are unsuitable for the transdermal delivery of mammalian cells. So far, the only reported MNs for cell delivery are hollow MNs, which a

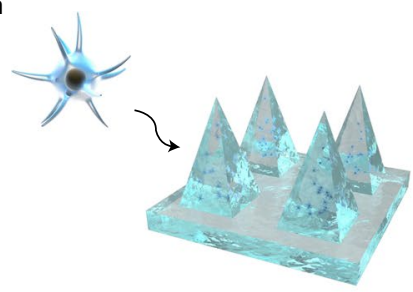

OVA-DC-loaded cryoMNs b

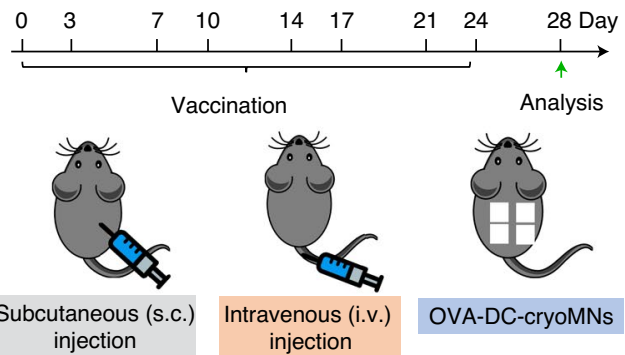

c

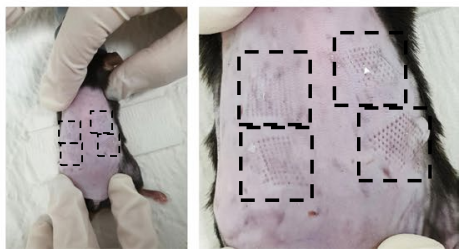

d
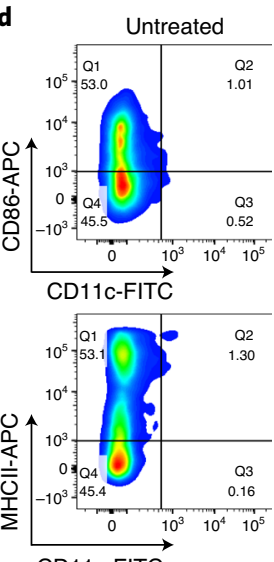

CD11C-FITC
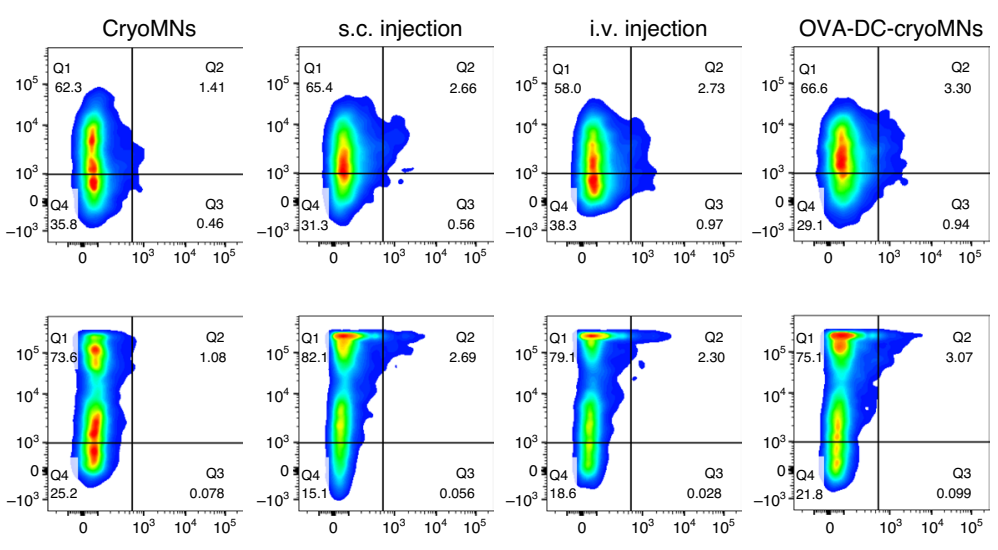

f

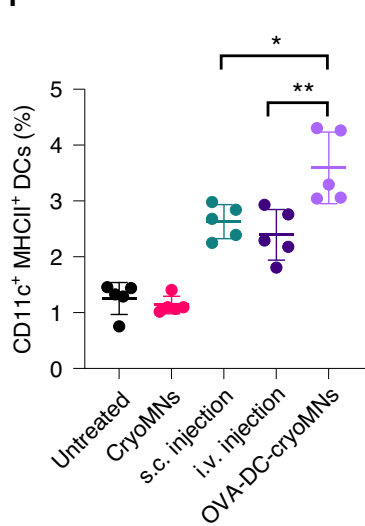

g

g Splenocyte proliferation

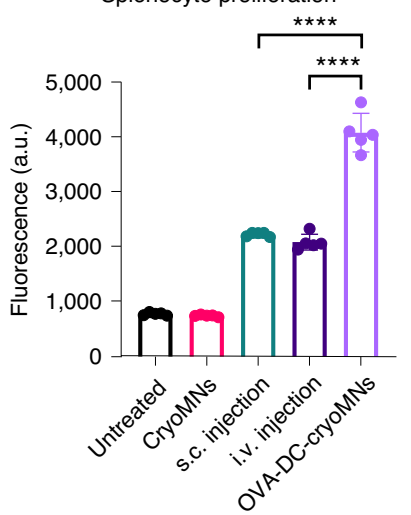

h

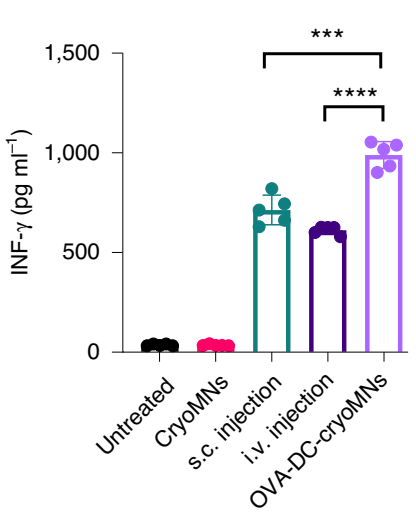

i
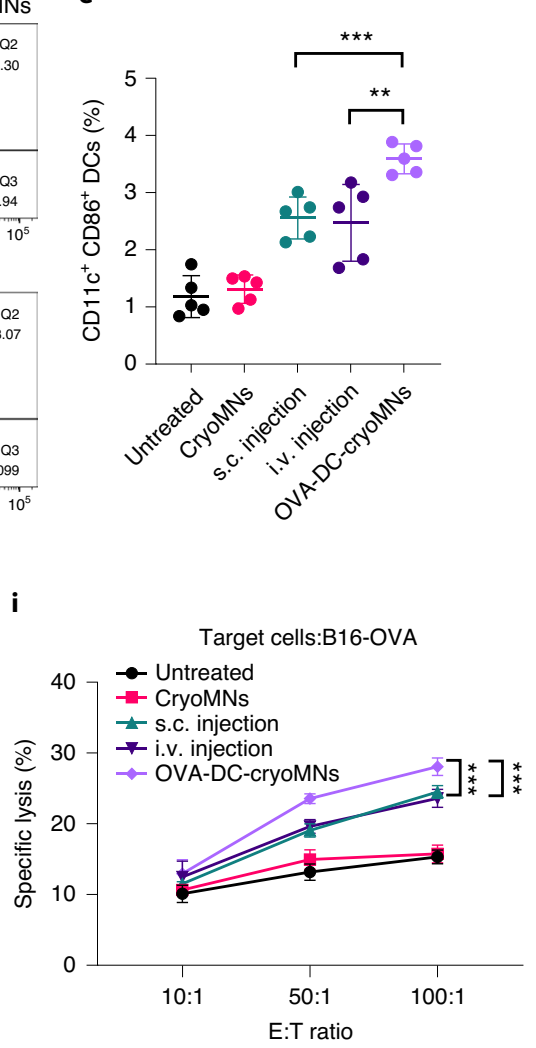
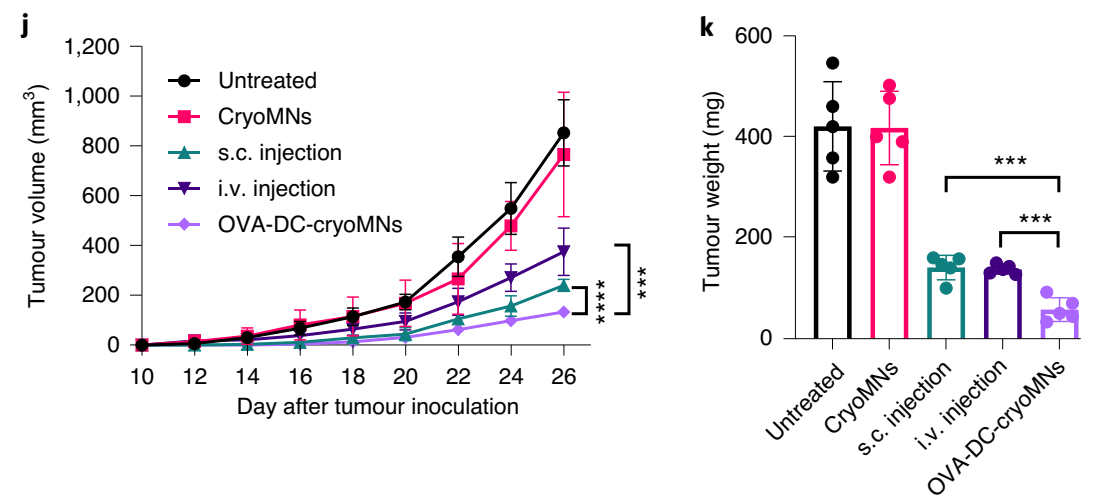

I

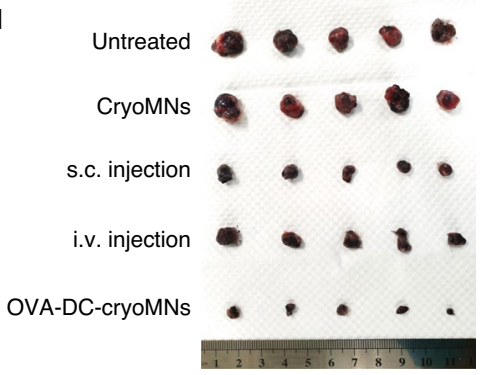


a

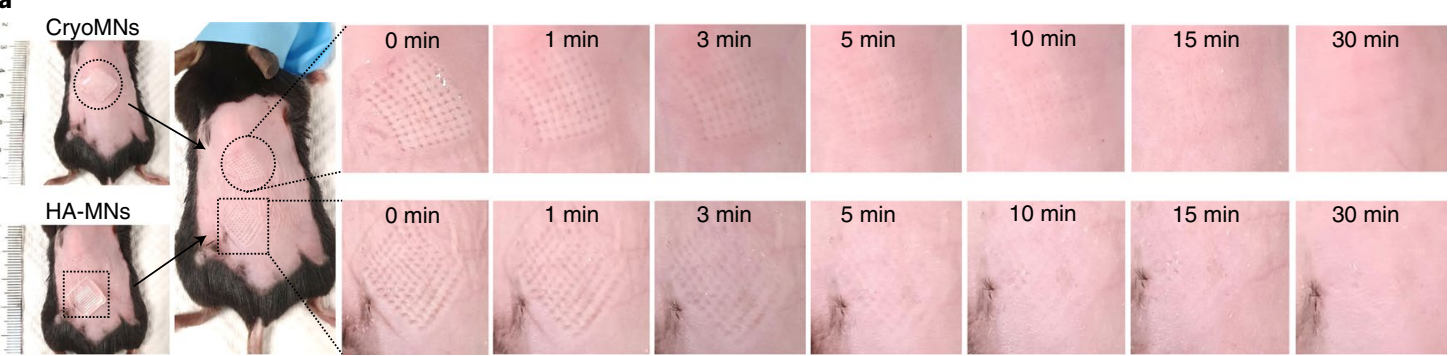

b

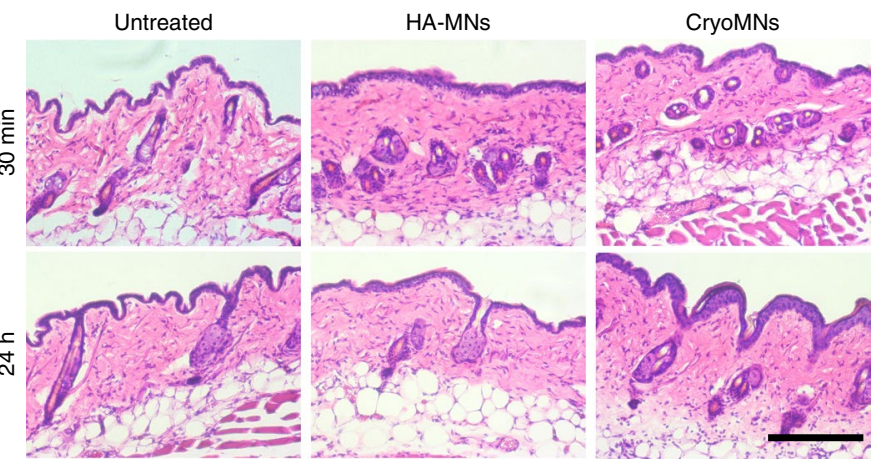

d

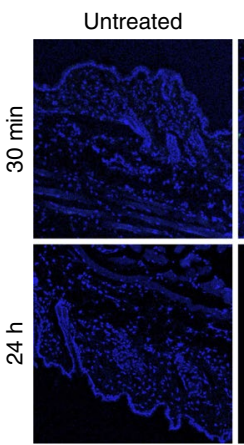

HA-MNs

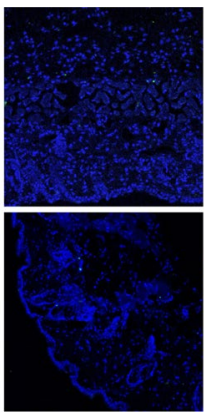

CryoMNs

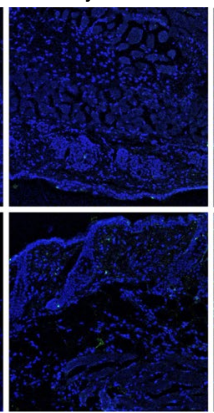

+DNase

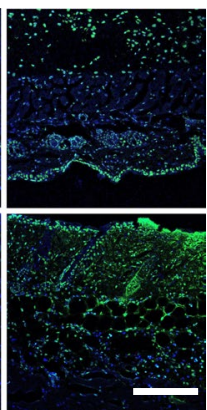

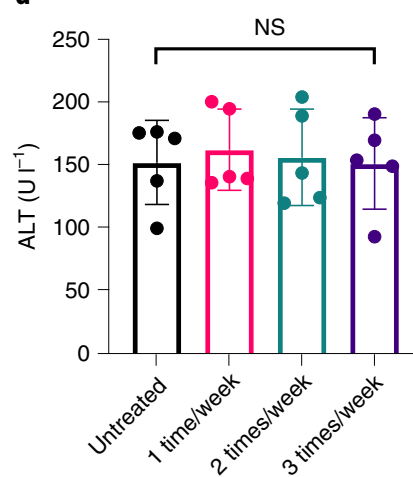

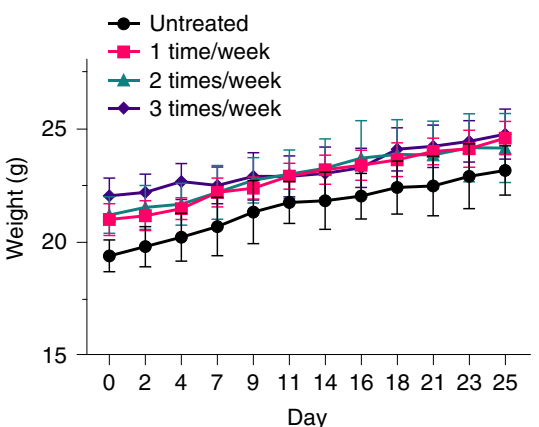

Fig. 6 | In vivo biocompatibility and safety studies of cryoMNs. a, Recovery of the skin after application of cryoMNs. MNs made of hyaluronic acid (HA-MNs) were used for comparison. The microholes gradually disappeared within 30 min. b, Representative images of H\&E staining of skin 30 min or $24 \mathrm{~h}$ after MN application. Scale bar, $200 \mu \mathrm{m}$. c, Representative images of skin stained with TUNEL assay (green) and Hoechst (blue) after MN application. Skin samples treated with DNase served as a positive control. Scale bar, $200 \mu \mathrm{m}$. d, Level of alanine aminotransferase (ALT) in livers of mice after administration of OVA-DC-loaded cryoMNs using different administration frequencies over a four-week period (untreated, one time per week, two times per week and three times per week). Data are presented as mean \pm s.d. ( $n=5$ independent animals). Two-tailed Student's $t$-test was used for comparing vaccination with OVA-DC-cryoMNs three times per week with the untreated group (NS, $P=0.9709$ ). e, Weight of mice administered OVA-DC-loaded cryoMNs at different administration frequencies. Data are presented as mean $\pm \mathrm{s} . \mathrm{d}$. ( $n=5$ independent animals). In $\mathbf{b}$ and $\mathbf{c}$, three images were taken for each group and all experiments were repeated independently twice, with similar results.

are employed for intradermal infusion of melanocytes into a specific skin layer ${ }^{37,38}$. However, hollow MNs have intrinsic limitations, including the plugging risk of narrow lumen and the breaking risk of a needle inside skin. Using cryoMNs can avoid these drawbacks. In addition, therapeutic cells can be directly encapsulated in cryoMNs during manufacture and remain viable after long-term storage (Supplementary Figs. 16, 20d and 21). Therefore, complex and redundant procedures, including cell harvesting and the preparation of cell infusing solution, can be circumvented during each administration. Moreover, cell delivery with cryoMNs does not need the assistance of additional equipment to provide infusion pressure, whereas such equipment is usually indispensable during the application of hollow MNs.

In the current design, DMSO was used as one of the raw materials for fabricating the cryoMNs. DMSO has been widely used in pharmaceutical formulations. It can be metabolized to dimethyl sulfone $\left(\mathrm{DMSO}_{2}\right)$ and dimethyl sulfide (DMS), and these metabolites can be readily excreted from the body, mainly via urine, without any residual accumulation. We evaluated the toxicity of DMSO in several types of skin cell and found that the cell viability remained greater than $80 \%$ after 24 -h incubation with $2.5 \%$ (vol/vol) DMSO (Supplementary Fig. 28). DMSO is generally regarded as non-toxic below $10 \%(\mathrm{vol} / \mathrm{vol})^{39}$. In addition, we calculated that the injected dose of DMSO per patch (100 arrays) was $\sim 1.1 \times 10^{-2} \mathrm{mg}$, which is much less than its median lethal dose $\left(\mathrm{LD}_{50}\right)^{40}$. Of note, this formulation of cryogenic medium is not unique and can be further developed by integrating state-of-the-art biotechnologies, such as new freezing protocols ${ }^{23}$ and cell encapsulation technology $y^{41}$. Our results showed that the application of cryoMNs did not cause localized or systematic abnormalities in mice (Fig. 6). In addition, two volunteers 
did not report any pain or harsh discomfort when placing cryoMNs on their finger skin without penetrating the skin (Supplementary Fig. 8b and Supplementary Video 3). Previous studies have demonstrated that cooling the skin can reduce patient discomfort during injection $^{42}$. Thus, cryoMNs could also function as local anaesthetics during administration. However, further detailed evaluation of safety in human subjects is still required to translate this technology to the bedside.

One potential clinical application of cryoMNs is the intradermal delivery of DC vaccines for cancer immunotherapy ${ }^{43,44}$. In the hospital setting, the protocol includes repeated cycles of blood collection from patients and the generation of $\mathrm{DCs}^{45,46}$. The whole process is time consuming and cost prohibitive, and there are batch-to-batch variations in DC quality. By contrast, DC vaccinations with cryoMNs only require one-time blood collection and generation of DCs, which are then packaged in cryoMNs as ready-to-use aliquots. This process would dramatically improve the practicability of DC vaccination. The intradermal route of administration of DC vaccines has been extensively considered superior to other administration routes, with stronger tumour-specific immune responses and fewer side effects ${ }^{44,47,48}$. However, intradermal injection is traditionally performed manually by the Mantoux technique, which is technically challenging ${ }^{49}$. Given the innate features of microdimensions, harnessing cryoMNs greatly simplifies the operation of intradermal injection. Here, we could easily perform intradermal DC vaccination in mice. Administration of the DC vaccine with cryoMNs elicited stronger antigen-specific immune responses and a more potent tumorigenic ability in mice than s.c. and i.v. injection (Fig. 5). We also found that the administration frequency and dosage influenced the immune responses (Supplementary Figs. 22 and 23), consistent with previous reports ${ }^{50,51}$. Other parameters, such as DC subsets, DC maturation and activation, tumour-associated antigens and cancer type, also affect vaccination outcomes ${ }^{52}$, which is worthy of further optimization to maximize therapeutic efficacy.

Although we used DC vaccination as a proof of concept, cryoMNs are suitable for packaging other types of therapeutic cell. For example, melanocyte-loaded cryoMNs can be used for the treatment of vitiligo ${ }^{8,9}$. Stem cell-loaded cryoMNs can be used to promote skin regeneration ${ }^{53}$. In addition to mammalian cells, cryoMNs can also be used to deliver probiotic bacteria ${ }^{54}$ or bacteriophages ${ }^{55}$ for the treatment of topical infection. Finally, this cryoMN technology is not limited to delivering live therapeutics. Similar to other types of $\mathrm{MN}$, cryoMNs can carry bioactive therapeutic agents, including proteins, peptides and vaccines. Due to the organic-free and low-temperature fabrication procedure, cryoMNs can maximally retain the bioactivity of those therapeutics.

Owing to the combination of MNs with cryopreservation technology, compared with the current standard of care, cryoMN technology not only achieves easy intradermal cell delivery with minimal invasiveness, but also simplifies the whole process of cell therapies by permitting multiple treatments with single donations. There are some translational considerations for this infant technology. First, the manufacture of cryoMNs is closely associated with the production of a clinically relevant number of therapeutic cells 5 . Reproducible manufacturing protocols of desirable cells for each therapy must be established. In addition, the materials for both the cryoMNs and cells have to meet the standards of the United States Food and Drug Administration (FDA) during the FDA approval process ${ }^{4}$. Second, because cryoMNs will be inserted into human skin, sterilization of cryoMN production is essential to prevent potential infections. CryoMNs can be manufactured under aseptic or sterile conditions. In addition, the feasibility of existing sterilization methods under low temperature is worthy of evaluation. Third, one cryoMN patch has limited cell capacity, which poses a critical issue if a large number of cells are required to induce potent therapeutic outcomes. It might be solved by the administration of multiple patches, but this might decrease patient compliance. An alternative solution is to increase the maximum cell capacity of a patch by either redesigning the cryoMNs with larger dimensions or enlarging the overall size of the patch with more MN arrays. Fourth, similar to COVID-19 mRNA vaccines, cryoMNs require ultracold shipping and storage conditions. Although traditional cryogenic equipment, such as $-80^{\circ} \mathrm{C}$ refrigerators and liquid nitrogen containers, are commonplace in most hospitals and research institutes, there is still an urgent need to develop new cryogenic equipment with low cost and high efficiency to facilitate distribution and decrease economic challenges, particularly in countries with limited resources. Furthermore, because cryoMNs will melt after being removed from their storage conditions, integrating cryoMNs with a handle or applicator will facilitate their easy manipulation and rapid penetration.

\section{Methods}

Fabrication of cell-loaded cryoMNs. The stainless-steel MNs were pre-designed and used as a master mould. To obtain the negative mould of the MNs, we replicated the master mould using 10:1 PDMS by pouring the liquid pre-polymer over the master $\mathrm{MN}$ structure, degassing in a vacuum oven for $10 \mathrm{~min}$ and curing the polymer at $70^{\circ} \mathrm{C}$ for $1 \mathrm{~h}$, and finally demoulding. The obtained PDMS mould was treated with $\mathrm{O}_{2}$ plasma and sterilized by ultraviolet exposure for $20 \mathrm{~min}$ before making cryoMNs. A 200- $\mu$ l volume of optimized cryogenic medium (PBS supplemented with $2.5 \%$ (vol/vol) DMSO and $100 \mathrm{mM}$ sucrose) was cast into the PDMS mould. Then PDMS mould was centrifuged at 4,000 r.p.m. for 3 min to allow the solution to fill up the needle cavities. The cells of interest were detached by trypsin and resuspended in cryogenic medium $\left(1 \times 10^{6} \mathrm{cells} \mathrm{ml}^{-1}\right)$. Next, $100 \mu \mathrm{l}$ of cell suspension $\left(1 \times 10^{5}\right.$ cells $)$ was added into the mould. The mould was placed aside for $20 \mathrm{~min}$ to allow the cells to fill up the needle cavities. The extra medium was removed from the base of the mould using a pipette and fresh cryogenic medium was added into the mould to fill up the base. The PDMS mould was held at $-20^{\circ} \mathrm{C}$ for $4 \mathrm{~h}$. Then cryoMNs were gently peeled off the PDMS mould, following by freezing at $-80^{\circ} \mathrm{C}$ for $2 \mathrm{~h}$ and at $-196^{\circ} \mathrm{C}$ (in liquid nitrogen) for $1 \mathrm{~h}$. The number of cells loaded in the MNs was calculated by subtracting the cell number in the removed medium from the cell number in the original cell suspension.

Morphology of cryoMNs. The cryoMNs were imaged by an optical microscope (Leica DVM6) and digital camera immediately after being removed from the liquid nitrogen. The melting behaviour of the cryoMNs was monitored by the digital camera. To visualize the cells (HaCaT) inside the cryoMNs, cell nuclei were stained with NucBlue Live ReadyProbes reagent (Invitrogen) before being loaded into the cryoMNs. The cryoMNs were placed into tissue culture plates and incubated in cold PBS for 30 min once being taken out of the liquid nitrogen. The samples were then visualized and imaged with a confocal microscope (LSM800, Carl Zeiss).

Evaluation of the mechanical strength of cryoMNs. The mechanical strength of the cryoMNs was examined using an Instron 5543 tensile meter. As shown in Supplementary Video 1, the cryoMNs were placed on a flat metal plate. The liquid nitrogen was used to provide a cooling environment for testing and to pre-cool both the probe and the metal plate. A vertical oriented force was applied perpendicularly to the MN tips, facing upward on a flat, rigid aluminium plate, using a 5-mm-diameter flat-head stainless-steel cylindrical probe (at a constant speed of $0.5 \mathrm{~mm} \mathrm{~min}^{-1}$ ). Four types of polymeric MN that are commonly used for transdermal drug delivery (PS-MNs, PLA-MNs, PCL-MNs and MeHA-CL-MNs ${ }^{15}$ ) were used for comparison.

Skin penetration ability of cryoMNs. CryoMNs loaded with RFP-HeLa cells were held at RT $\left(24^{\circ} \mathrm{C}\right)$ for a designated time $(0 \mathrm{~s}, 10 \mathrm{~s}, 20 \mathrm{~s}, 30 \mathrm{~s}, 40 \mathrm{~s}, 50 \mathrm{~s}$ and $60 \mathrm{~s})$ after being taken out of liquid nitrogen. The cryoMNs were inserted into porcine skin (fresh porcine cadaver skin was purchased from the local supermarket) by thumb press. The skin tissue was further fixed with $4 \%$ paraformaldehyde and stained with $\mathrm{H} \& \mathrm{E}$ for histological analysis. To visualize the cell distribution in the skin, the skin tissue was directly processed for cryo-sectioning and observed under a fluorescence microscope. All images were analysed by ImageJ software (version $1.53 \mathrm{a}$, no plugin used)

Viability and proliferation of cells. Cell-loaded cryoMNs were placed into the 48-well plates without supplementation of culture medium and the live/dead cell viability assay was conducted according to the protocol provided by the manufacturers. All fluorescent images were analysed by ImageJ software (version 1.53a, no plugin used). Quantitative data for cell viability were calculated according to live (green)/dead (red) staining by dividing the number of living cells (green) by the total number of cells. In the proliferation assay, cell-loaded cryoMNs were transferred into 48-well plates with supplementation of culture medium. Meanwhile, the same types of cell were seeded into the plates with a density of 
$1 \times 10^{5}$ cells per well, for use as a positive control. The cells were cultured and the alamarBlue assay was conducted at days 1, 3 and 6 according to the protocol provided by the manufacturer. Briefly, alamarBlue reagent was added into each well to a final concentration of $10 \%(\mathrm{vol} / \mathrm{vol})$ and the fluorescence intensity (excitation $540 \mathrm{~nm}$, emission $580 \mathrm{~nm}$ ) was measured after a 4 -h incubation by using a plate reader (SpectraMax M5, Molecular Devices). Quantification of cell proliferation was obtained by dividing the fluorescence intensity at each designated culture time by the fluorescence intensity after one-day culture. In addition, cells were visualized by a phase contrast microscope at each designated time point.

Transdermal delivery of RFP-HeLa cells with cryoMNs on NOD/SCID mice. All mice used in the present study were housed at $20-24^{\circ} \mathrm{C}$ and $30-70 \%$ humidity. The light/dark cycle of the holding room was $12 \mathrm{~h} / 12 \mathrm{~h}$ from 8:00 to 20:00. All mice were fed with food and water ad libitum. Non-obese diabetic/severe combined immunodeficiency (NOD/SCID) mice experiments were performed in accordance with ethical approval by the Nanyang Technological University Institutional Animal Care and Use Committee (NTU-IACUC) under protocol A18001. The NOD/SCID mice (male, 6-8 weeks, 20-25 g) were purchased from InVivos. The mice were dehaired under anaesthesia before use. Blank cryoMNs and RFP-HeLa-loaded cryoMNs were applied on the left and right flanks of mouse backs for $5 \mathrm{~min}$. To confirm cell survival after delivery into the mice skin, the $\mathrm{MN}$-treated mice were imaged under an in vivo imaging system (IVIS, Spectrum, Perkin Elmer) using a filter set (excitation $570 \mathrm{~nm}$, emission $680 \mathrm{~nm}$ ), 2F/stop and 13.6- $\mathrm{cm}$ field of view for RFP imaging at the designated times (days $0,1,3,7$ and 14). The exposure time was $15 \mathrm{~s}$. Quantitative analysis of the RFP fluorescence intensity was conducted by measuring the average radiant efficiency (photons $\mathrm{s}^{-1} \mathrm{~cm}^{-2} \mathrm{sr}^{-1} \mu \mathrm{W}^{-1}$ ) in regions of interest (ROIs) that were placed on the application site of cryoMNs. We subtracted the ROI value acquired from untreated skin on the same mouse. The data are normalized to the fluorescence intensity at day 0 .

Vaccination with OVA-DC-cryoMNs. Experiments with C57BL/6 mice (female, 6-8 weeks, $20-25 \mathrm{~g}$ ) were performed in accordance with ethical approval by the Animal Research Ethics Sub-Committee of City University of Hong Kong (internal ref. A-0493). The mice were randomly distributed into one of the following five treatment groups: untreated, cryoMNs, s.c. injection of OVA-DCs, i.v. injection of OVA-DCs (injection through the tail vein without saline flush), OVA-DC-cryoMNs. Each group of mice received vaccination on days $0,3,7,10$, $14,17,21$ and 24. During each vaccination, four patches of OVA-DC-cryoMNs were applied to deliver $4 \times 10^{5}$ OVA-DCs per mouse. For s.c. and i.v. injection, $2.8 \times 10^{5}$ OVA-DCs was injected each time for each mouse (that is, $70 \%$ of cells applied for cryoMN vaccination). At day 28 , lymph nodes and major organs (heart, lung, liver, spleen and kidney) were excised from the mice. Lymph nodes and partial spleen were homogenized by grinding with the end of a sterile syringe and processed into a single cell suspension. To study the homing of mature DCs to the lymph nodes, cells extracted from the lymph nodes were stained with FITC-conjugated CD11c antibody staining (1:200) to analyse DC infiltration, and APC-conjugated CD86 antibody (1:300) and APC-conjugated MHCII antibody $(1: 100)$ to evaluate DC activation and maturation, respectively. The stained cells were measured by a flow cytometer (BD Biosciences, BD FACSDiva software v 8.0) and were analysed by FlowJo software (TreeStar, version 10.5.3)

A splenocytes proliferation and CTL assay were then performed. Splenocytes $\left(5 \times 10^{5}\right.$ per well) were seeded in a 96 -well plate and restimulated with $50 \mu \mathrm{g} \mathrm{ml}^{-1}$ OVA for two days. Splenocytes proliferation was evaluated using an alamarBlue cell viability assay according to the protocol provided by the manufacturer. The production of IFN- $\gamma$ in culture supernatants was measured by a mouse IFN- $\gamma$ enzyme-linked immunosorbent assay kit. The CTL assay was conducted following the manufacturer's protocol (CyQUANT LDH cytotoxicity assay kit). Briefly, the B16 melanoma cell line transfected with ovalbumin (B16-OVA; target cells) was loaded with the fluorescence enhancing ligand. Splenocytes (effector cells) and B16-OVA (target cells) were then co-cultured in U-bottomed 96-well plates with cell number ratios of 10:1. 50:1 and 100:1. After incubation for $2 \mathrm{~h}$ at $37^{\circ} \mathrm{C}$, the lysed target cells were quantified. The major organs were cryosectioned and H\&E stained to analyse for any organ damage after the vaccination process.

To evaluate the antitumour efficacy of the OVA-DC-cryoMNs vaccination, melanoma tumour models were developed on vaccinated C57BL/6 mice through s.c. injection of $2 \times 10^{5} \mathrm{~B} 16$-OVA cells in the right flank at day 28 . The tumour volume was monitored every other day after cell inoculation by a digital caliper. The tumour volume (cubic millimetre) was calculated as $1 / 2 \times$ long diameter $\times$ (short diameter $)^{2}$. The tumour tissues and major organs were collected 26 days after cell inoculation following mice euthanasia, and the weight of the tumours was measured.

In vivo skin compatibility and safety evaluation. The cryoMNs patches and HA-MNs patches (as control) were applied to each flank of the dorsal skin of mice and removed after $10 \mathrm{~min}$. To visualize skin resealing and irritation, the treated sites were imaged with a digital camera at pre-designated time points. For histological analysis, the treated sites were collected and processed for cryo-sectioning, then H\&E and TUNEL staining were performed on the skin sections.
Statistical analysis. All experiments used biological replicates that consisted of cells in non-repeated, independent cell culture wells or tissue samples from different animals, unless specified otherwise. Quantitative data are represented as mean \pm s.d. Statistical analysis was performed by using two-tailed Student's $t$-test or original one-way analysis of variance. $P<0.05$ was considered statistically significant $\left({ }^{\star} P<0.05,{ }^{* *} P<0.01,{ }^{* * *} P<0.001,{ }^{* * * *} P<0.0001\right)$. GraphPad Prism 8.3 was used for data analysis. Microsoft Excel 2019 was used for calculating the exact $P$ value when $P<0.0001$

Additional experiments. Materials, cell culture, optimization of cryogenic medium, generation and stimulation of bone marrow-derived dendritic cells and optimization of vaccination with OVA-DC-cryoMNs are available in the Supplementary Information

Reporting Summary. Further information on research design is available in the Nature Research Reporting Summary linked to this article.

\section{Data availability}

The main data supporting the results in this study are available within the paper and its Supplementary Information. The raw and analysed datasets generated during the study are too large to be publicly shared, yet they are available for research purposes from the corresponding author on reasonable request.

Received: 18 November 2018; Accepted: 26 March 2021; Published online: 3 May 2021

\section{References}

1. Pupovac, A. et al. Toward immunocompetent 3D skin models. Adv. Healthc. Mater. 7, e1701405 (2018).

2. MacNeil, S. Progress and opportunities for tissue-engineered skin. Nature 445, 874-880 (2007)

3. Dabrowska, A. K. et al. The relationship between skin function, barrier properties and body-dependent factors. Skin Res. Technol. 24, 165-174 (2018)

4. Facklam, A. L., Volpatti, L. R. \& Anderson, D. G. Biomaterials for personalized cell therapy. Adv. Mater. 32, e1902005 (2020).

5. Aijaz, A. et al. Biomanufacturing for clinically advanced cell therapies. Nat. Biomed. Eng. 2, 362-376 (2018).

6. Fuchs, E. Skin stem cells in silence, action and cancer. Stem Cell Rep. 10, 1432-1438 (2018).

7. Mavilio, F. et al. Correction of junctional epidermolysis bullosa by transplantation of genetically modified epidermal stem cells. Nat. Med. 12, 1397-1402 (2006).

8. Fan, S. M. et al. Preclinical evaluation of melanocyte transplantation by chitosan-based melanocyte spheroid patch to skin prepared by controlled sunburn blistering. J. Biomed. Mater. Res. B Appl. Biomater. 106, 2535-2543 (2018).

9. Lotti, T., Gori, A., Zanieri, F., Colucci, R. \& Moretti, S. Vitiligo: new and emerging treatments. Dermatol. Ther. 21, 110-117 (2008).

10. Saxena, M. \& Bhardwaj, N. Re-emergence of dendritic cell vaccines for cancer treatment. Trends Cancer 4, 119-137 (2018).

11. Banchereau, J. \& Palucka, A. K. Dendritic cells as therapeutic vaccines against cancer. Nat. Rev. Immunol. 5, 296-306 (2005).

12. Mooney, D. J. \& Vandenburgh, H. Cell delivery mechanisms for tissue repair. Cell Stem Cell 2, 205-213 (2008).

13. Bhatnagar, S., Dave, K. \& Venuganti, V. V. K. Microneedles in the clinic. J. Control. Release 260, 164-182 (2017).

14. Ye, Y., Yu, J., Wen, D., Kahkoska, A. R. \& Gu, Z. Polymeric microneedles for transdermal protein delivery. Adv. Drug Deliv. Rev. 127, 106-118 (2018).

15. Chang, H. et al. A swellable microneedle patch to rapidly extract skin interstitial fluid for timely metabolic analysis. Adv. Mater. 29, 1702243 (2017)

16. Than, A. et al. Self-implantable double-layered micro-drug-reservoirs for efficient and controlled ocular drug delivery. Nat. Commun. 9, 4433 (2018).

17. Chang, H., Zheng, M., Chew, S. W. T. \& Xu, C. Advances in the formulations of microneedles for manifold biomedical applications. Adv. Mater. Technol. 5, 1900552 (2020).

18. Zhang, Y. et al. Thrombin-responsive transcutaneous patch for auto-anticoagulant regulation. Adv. Mater. 29, 1604043 (2017).

19. Petrovic, J. J. Review: Mechanical properties of ice and snow. J. Mater. Sci. 38, $1-6(2003)$.

20. Crowe, J. H. \& Crowe, L. M. Preservation of mammalian cells-learning nature's tricks. Nat. Biotechnol. 18, 145-146 (2000).

21. Janz Fde, L. et al. Evaluation of distinct freezing methods and cryoprotectants for human amniotic fluid stem cells cryopreservation. J. Biomed. Biotechnol. 2012, 649353 (2012).

22. Matsumura, K. \& Hyon, S. H. Polyampholytes as low toxic efficient cryoprotective agents with antifreeze protein properties. Biomaterials 30, 4842-4849 (2009). 
23. Huang, $\mathrm{H}$. et al. Predehydration and ice seeding in the presence of trehalose enable cell cryopreservation. ACS Biomater. Sci. Eng. 3, 1758-1768 (2017).

24. Rodrigues, J. P. et al. Evaluation of trehalose and sucrose as cryoprotectants for hematopoietic stem cells of umbilical cord blood. Cryobiology 56, 144-151 (2008).

25. Kim, J. D., Kim, M., Yang, H., Lee, K. \& Jung, H. Droplet-born air blowing: novel dissolving microneedle fabrication. J. Control. Release 170, 430-436 (2013).

26. Abd, E. et al. Skin models for the testing of transdermal drugs. Clin. Pharmacol. 8, 163-176 (2016).

27. Kietzmann, M., Lubach, D. \& Heeren, H. J. The mouse epidermis as a model in skin pharmacology: influence of age and sex on epidermal metabolic reactions and their circadian rhythms. Lab Anim. 24, 321-327 (1990).

28. Wang, H. \& Mooney, D. J. Biomaterial-assisted targeted modulation of immune cells in cancer treatment. Nat. Mater. 17, 761-772 (2018).

29. Saxena, M., Balan, S., Roudko, V. \& Bhardwaj, N. Towards superior dendritic-cell vaccines for cancer therapy. Nat. Biomed. Eng. 2, 341-346 (2018).

30. Sullivan, S. P. et al. Dissolving polymer microneedle patches for influenza vaccination. Nat. Med. 16, 915-920 (2010)

31. $\mathrm{Yu}$, J. et al. Hypoxia and $\mathrm{H}_{2} \mathrm{O}_{2}$ dual-sensitive vesicles for enhanced glucose-responsive insulin delivery. Nano Lett. 17, 733-739 (2017).

32. Petrof, G., Abdul-Wahab, A. \& McGrath, J. A. Cell therapy in dermatology. Cold Spring Harb. Perspect. Med 4, a015156 (2014).

33. Ortiz-Urda, S. et al. Injection of genetically engineered fibroblasts corrects regenerated human epidermolysis bullosa skin tissue. J. Clin. Invest. 111, 251-255 (2003).

34. Chen, F., Yan, Q., Yu, Y. \& Wu, M. X. BCG vaccine powder-laden and dissolvable microneedle arrays for lesion-free vaccination. J. Control. Release 255, 36-44 (2017)

35. Hiraishi, Y. et al. Bacillus Calmette-Guérin vaccination using a microneedle patch. Vaccine 29, 2626-2636 (2011).

36. Tang, J. et al. Cardiac cell-integrated microneedle patch for treating myocardial infarction. Sci. Adv. 4, eaat9365 (2018).

37. Iliescu, F. S., Teo, J. C. M., Vrtacnik, D., Taylor, H. \& Iliescu, C. Cell therapy using an array of ultrathin hollow microneedles. Microsyst. Technol. 24, 2905-2912 (2017)

38. Gualeni, B. et al. Minimally invasive and targeted therapeutic cell delivery to the skin using microneedle devices. Br. J. Dermatol. 178, 731-739 (2018).

39. Galvao, J. et al. Unexpected low-dose toxicity of the universal solvent DMSO. FASEB J. 28, 1317-1330 (2014).

40. Smith, E. R., Hadidian, Z. \& Mason, M. M. The single- and repeated-dose toxicity of dimethyl sulfoxide. Ann. N. Y. Acad. Sci. 141, 96-109 (1967)

41. Youn, W. et al. Cytoprotective encapsulation of individual Jurkat T cells within durable $\mathrm{TiO}_{2}$ shells for T-cell therapy. Angew. Chem. Int. Ed. 56, 10702-10706 (2017)

42. Nestor, M. S., Ablon, G. R. \& Stillman, M. A. The use of a contact cooling device to reduce pain and ecchymosis associated with dermal filler injections. J. Clin. Aesthet. Dermatol. 3, 29-34 (2010).

43. Santos, P. M. \& Butterfield, L. H. Dendritic cell-based cancer vaccines. J. Immunol. 200, 443-449 (2018).

44. Seyfizadeh, N., Muthuswamy, R., Mitchell, D. A., Nierkens, S. \& Seyfizadeh, N. Migration of dendritic cells to the lymph nodes and its enhancement to drive anti-tumor responses. Crit. Rev. Oncol. Hematol. 107, 100-110 (2016).

45. Shinde, P., Khan, N., Melinkeri, S., Kale, V. \& Limaye, L. Freezing of dendritic cells with trehalose as an additive in the conventional freezing medium results in improved recovery after cryopreservation. Transfusion 59, 686-696 (2019).

46. Zhou, Q. et al. Mature dendritic cell derived from cryopreserved immature dendritic cell shows impaired homing ability and reduced anti-viral therapeutic effects. Sci. Rep. 6, 39071 (2016).
47. Edele, F. et al. Efficiency of dendritic cell vaccination against B16 melanoma depends on the immunization route. PLOS ONE 9 e105266 (2014).

48. Fong, L., Brockstedt, D., Benike, C., Wu, L. \& Engleman, E. G. Dendritic cells injected via different routes induce immunity in cancer patients. J. Immunol. 166, 4254-4259 (2001).

49. Laurent, P. E. et al. Evaluation of the clinical performance of a new intradermal vaccine administration technique and associated delivery system. Vaccine 25, 8833-8842 (2007)

50. Lesterhuis, W. J. et al. Dendritic cell vaccines in melanoma: from promise to proof? Crit. Rev. Oncol. Hematol. 66, 118-134 (2008).

51. Figdor, C. G., de Vries, I. J., Lesterhuis, W. J. \& Melief, C. J. Dendritic cell immunotherapy: mapping the way. Nat. Med. 10, 475-480 (2004).

52. Wculek, S. K. et al. Dendritic cells in cancer immunology and immunotherapy. Nat. Rev. Immunol. 20, 7-24 (2020).

53. Hirsch, T. et al. Regeneration of the entire human epidermis using transgenic stem cells. Nature 551, 327-332 (2017).

54. Hwang, I. Y. et al. Engineered probiotic Escherichia coli can eliminate and prevent Pseudomonas aeruginosa gut infection in animal models. Nat. Commun. 8, 15028 (2017).

55. Ryan, E. M., Gorman, S. P., Donnelly, R. F. \& Gilmore, B. F. Recent advances in bacteriophage therapy: how delivery routes, formulation, concentration and timing influence the success of phage therapy. J. Pharm. Pharmacol. 63, 1253-1264 (2011)

\section{Acknowledgements}

C.X. acknowledges funding support from the City University of Hong Kong (no. 9610472), the General Research Fund (GRF) from the University Grant Committee of Hong Kong (UGC) Research Grant Council (RGC) (no. 9042951) and NSFC/RGC Joint Research Scheme (N_CityU118/20). C.X. also appreciates constructive discussion with R. Langer (Massachusetts Institute of Technology) for the vaccination experiments.

\section{Author contributions}

H.C. and C.X. conceived the project and designed the experiments. H.C., S.W.T.C., M.Z., D.C.S.L., C.W., X.N., A.T. and Y.M. performed the experiments. H.C., S.W.T.C., M.Z., M.C. and D.C.S.L. collected data. H.C. and C.X. were primarily responsible for the data analysis and interpretation. H.C. prepared the figures. H.L. helped design the animal experiments. H.C. and C.X. wrote the manuscript. H.L., P.C., K.P., D.W. and P.S. provided valuable input for the write-up. All authors discussed the results and reviewed the manuscript.

\section{Competing interests}

H.C., P.C. and C.X. are inventors in a patent application that has been filed based on the data in this manuscript.

\section{Additional information}

Supplementary information The online version contains supplementary material available at https://doi.org/10.1038/s41551-021-00720-1.

Correspondence and requests for materials should be addressed to C.X.

Peer review information Nature Biomedical Engineering thanks Michael Mitchell and the other, anonymous, reviewer(s) for their contribution to the peer review of this work.

Reprints and permissions information is available at www.nature.com/reprints.

Publisher's note Springer Nature remains neutral with regard to jurisdictional claims in published maps and institutional affiliations.

(C) The Author(s), under exclusive licence to Springer Nature Limited 2021 


\title{
natureresearch
}

Corresponding author(s): Chenjie Xu

Last updated by author(s): Jan 30, 2021

\section{Reporting Summary}

Nature Research wishes to improve the reproducibility of the work that we publish. This form provides structure for consistency and transparency in reporting. For further information on Nature Research policies, see Authors \& Referees and the Editorial Policy Checklist.

\section{Statistics}

For all statistical analyses, confirm that the following items are present in the figure legend, table legend, main text, or Methods section.

n/a Confirmed

$\bigotimes$ The exact sample size $(n)$ for each experimental group/condition, given as a discrete number and unit of measurement

$\bigotimes$ A statement on whether measurements were taken from distinct samples or whether the same sample was measured repeatedly

The statistical test(s) used AND whether they are one- or two-sided

Only common tests should be described solely by name; describe more complex techniques in the Methods section.

$\searrow \square$ A description of all covariates tested

\ $\square$ A description of any assumptions or corrections, such as tests of normality and adjustment for multiple comparisons

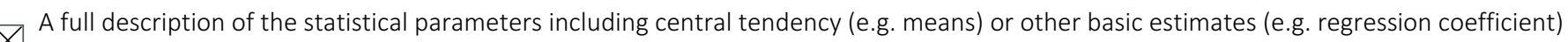

$\triangle$ AND variation (e.g. standard deviation) or associated estimates of uncertainty (e.g. confidence intervals)

For null hypothesis testing, the test statistic (e.g. $F, t, r$ ) with confidence intervals, effect sizes, degrees of freedom and $P$ value noted

Give $P$ values as exact values whenever suitable.

Х $\square$ For Bayesian analysis, information on the choice of priors and Markov chain Monte Carlo settings

Х $\square$ For hierarchical and complex designs, identification of the appropriate level for tests and full reporting of outcomes

Х $\square$ Estimates of effect sizes (e.g. Cohen's $d$, Pearson's $r$ ), indicating how they were calculated

Our web collection on statistics for biologists contains articles on many of the points above.

\section{Software and code}

\section{Policy information about availability of computer code}

Data collection

\section{ImageJ 1.53a}

BD FACSDiva' ${ }^{\mathrm{TM}}$ Software v8.0 (BD Biosciences, USA)

Leica TCS SP8 Confocal Microscope System

Molecular Devices SpectraMax M5e Microplate Reader

Data analysis

\author{
Microsoft Excel 2019 \\ ImageJ 1.53a \\ FlowJo 10.5.3
}

GraphPad Prism 8.3

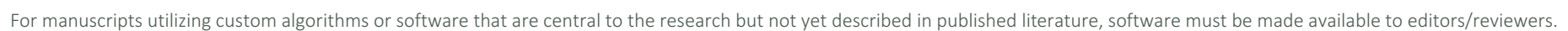
We strongly encourage code deposition in a community repository (e.g. GitHub). See the Nature Research guidelines for submitting code \& software for further information.

\section{Data}

Policy information about availability of data

All manuscripts must include a data availability statement. This statement should provide the following information, where applicable:

- Accession codes, unique identifiers, or web links for publicly available datasets

- A list of figures that have associated raw data

- A description of any restrictions on data availability

The main data supporting the results in this study are available within the paper and its Supplementary Information. The raw and analysed datasets generated during the study are too large to be publicly shared, yet they are available for research purposes from the corresponding authors on reasonable request. 
Please select the one below that is the best fit for your research. If you are not sure, read the appropriate sections before making your selection. \Life sciences Behavioural \& social sciences Ecological, evolutionary \& environmental sciences

For a reference copy of the document with all sections, see nature.com/documents/nr-reporting-summary-flat.pdf

\section{Life sciences study design}

All studies must disclose on these points even when the disclosure is negative.

Sample size No statistical methods were used to determine sample size. Sample sizes were determined on the basis of previous experimental experience (Refs: Adv. Mater. 2017, 29: 1702243; Nat. Commun. 2018, 9: 4433; Adv. Healthcare Mater. 2020, 9: e1901683). In vitro experiments were performed with at least 3 biologically independent samples. All in vivo experiments were performed with at least 3 independent animals. Sample sizes were sufficient to perform statistical analyses.

Data exclusions No data were excluded from the analyses.

Replication All in vitro and in vivo experiments were carried out with at least 3 biological replicates for each experimental group.

Randomization All samples and organisms were randomly allocated into experimental groups.

Blinding No formal blinding was used. The investigators were unblinded during the administration and characterization of the cryomicroneedles, to minimize improper operation during their handling. For the animal experiments, the investigators were blinded to data collection and analysis.

\section{Reporting for specific materials, systems and methods}

We require information from authors about some types of materials, experimental systems and methods used in many studies. Here, indicate whether each material, system or method listed is relevant to your study. If you are not sure if a list item applies to your research, read the appropriate section before selecting a response.

Materials \& experimental systems

\begin{tabular}{l|l}
\hline$n / a$ & Involved in the study \\
\hline & $\bigotimes$ Antibodies \\
$\square$ & $\square$ Eukaryotic cell lines \\
$\square$ & $\square$ Palaeontology \\
$\square$ Clinical data
\end{tabular}

\begin{tabular}{l|l} 
Methods \\
\hline n/a & Involved in the study \\
$X$ & $\square$ ChIP-seq \\
$\square$ & $\square$ Flow cytometry \\
$\square$ & $\square$ MRI-based neuroimaging
\end{tabular}

\section{Antibodies}

Antibodies used Anti-mouse MHC Class II antibody (APC-conjugated, AF6-120.1, Catalog \# 17-5320-82, dilution: 1:100) and anti-mouse CD86 antibody (APC-conjugated ,GL1, Catalog \# 17-0862-82, dilution: 1:300) were purchased from ThermoFisher Scientific (USA) Anti-Mouse CD11C Antibody (FITC conjugated, Clone: N418, Catalog \# 60002FI, dilution 1: 200) was purchased from the StemCell Pte. Ltd. (Singapore).

Validation

All antibodies were validated by the manufacturers.

https://www.stemcell.com/anti-mouse-cd11c-antibody-clone-n418.html

https://www.thermofisher.com/antibody/product/CD86-B7-2-Antibody-clone-GL1-Monoclonal/17-0862-82

https://www.thermofisher.com/antibody/product/MHC-Class-II-I-Ab-Antibody-clone-AF6-120-1-Monoclonal/17-5320-82

\section{Eukaryotic cell lines}

Policy information about cell lines

Cell line source(s)

Human immortalized (HaCaT) and Normal dermal (NDFs) were obtained from CellResearch Corporation Pte Ltd, Singapore. Human Hela-Red fluorescent protein (RFP) stable cell line (RFP-Hela) was purchased from GenTarget Inc, San Diego, USA. Human epidermal melanocytes from darkly pigmented donor was purchased from ThermoFisher Scientific, USA Human mesenchymal stem cells (MSCs) was purchased from Lonza. Human Peripheral Blood CD8+CD45RA+ T Cells was purchased from StemCell Pte. Ltd. ,Singapore

The B16 melanoma cell line transfected with ovalbumin (B16-OVA) was gifted by the lab of professor Jiandong Huang from School of Biomedical Sciences, The University of Hong Kong, Hong Kong, and professor Haiyan Liu from Life Sciences Institute 
and Department of Microbiology and Immunology, National University of Singapore, Singapore.

Bone marrow derived dendritic cells were derived from C57BL/6 according to protocol (Madaan, A. et al. J. Biol. Meth., 1, 1 (2014)).

Authentication

Mycoplasma contamination

Commonly misidentified lines (See ICLAC register)
Each cell line used was morphologically confirmed according to the information provided by their vendors.

Bone marrow derived dendritic cells were confirmed by checking the morphology and the expression of CD11c, according to previous literature (Madaan, A. et al. J. Biol. Meth. 1, 1 (2014)).

All cell lines tested negative for mycoplasma.

No commonly misidentified cell lines were used.

\section{Animals and other organisms}

Policy information about studies involving animals; ARRIVE guidelines recommended for reporting animal research

Laboratory animals

The NOD/SCID mice (male, 6-8 weeks, 20-25 g) were purchased from InVivos Co., Ltd. (Singapore).

The C57BL/6 mice (male, 6-8 weeks, 20-25 g) were purchased from InVivos Co., Ltd. (Singapore).

The C57BL/6 mice (female, 6-8 weeks, 20-25 g) were purchased from the Laboratory Animal Research Unit (LARU), City

University of Hong Kong (Hong Kong).

Wild animals

The study did not involve wild animals.

Field-collected samples

The study did not involve samples collected from the field.

Ethics oversight

The animal experiments were performed under ethical approval by the Nanyang Technological University Institutional Animal Care and Use Committee (NTU-IACUC; protocol A18001) and by the Animal Research Ethics Sub-Committee of the City University of Hong Kong (Internal Ref: A-0493).

Note that full information on the approval of the study protocol must also be provided in the manuscript.

\section{Flow Cytometry}

\section{Plots}

Confirm that:

Х The axis labels state the marker and fluorochrome used (e.g. CD4-FITC).

Х The axis scales are clearly visible. Include numbers along axes only for bottom left plot of group (a 'group' is an analysis of identical markers).

Х All plots are contour plots with outliers or pseudocolor plots.

Х A numerical value for number of cells or percentage (with statistics) is provided.

\section{Methodology}

Sample preparation

Instrument

Lymph nodes were harvested into a tissue-culture dish, and teased apart into a single-cell suspension by pressing with the plunger of a syringe. Cells were collected in $10 \mathrm{~mL}$ of Flow Cytometry Staining Buffer, and the cell-suspension was passed through a cell strainer to eliminate clumps and debris. The cell suspension was collected in a conical tube. The cell suspension was centrifuged $4-5$ minutes $(300-400 \times g)$ at $4^{\circ} \mathrm{C}$, and the supernatant was discarded. The cell pellet was resuspended, and the final cell concentration made was $2 \times 10^{\wedge} 6 / \mathrm{mL}$ at 100 ul. The cells were then stained with FITC-conjugated CD11c antibody for analysing DC infiltration, and with APC-conjugated CD86 antibody and APC-conjugated $\mathrm{MHCI}$ antibody for evaluating DC activation and maturation, respectively.

BD LSRFortessa ${ }^{\mathrm{TM}} \mathrm{X}$-20 cell analyzer (BD Biosciences, USA).

BD Biosciences benchtop Flow Cytometer.

Software

BD FACSDiva Software v8.0 (BD Biosciences, USA) for data collection. FlowJo v10.5.3 software (FlowJo LLC, Becton Dickinson, USA) for processing and analysis.

Cell population abundance

Gating strategy

\section{No sorting was performed.}

Generally, cells were first gated on FSC/SSC. Singlet cells were usually gated using FSC-H and FSC-A. CD11c \& CD86 gating and $\mathrm{CD} 11 \mathrm{c} \& \mathrm{MHCll}$ gating were performed.

Х Tick this box to confirm that a figure exemplifying the gating strategy is provided in the Supplementary Information. 